\title{
Symmetry of convex sets and its applications to the extremal ellipsoids of convex bodies
}

\author{
Osman Güler $\quad$ Filiz Gürtuna *
}

February 2011

\begin{abstract}
A convex body $K$ in $\mathbb{R}^{n}$ has around it a unique circumscribed ellipsoid $\mathrm{CE}(K)$ with minimum volume, and within it a unique inscribed ellipsoid $\operatorname{IE}(K)$ with maximum volume. The modern theory of these ellipsoids is pioneered by Fritz John in his seminal 1948 paper. This paper has two, related goals. First, we investigate the symmetry properties of a convex body by studying its (affine) automorphism group $\operatorname{Aut}(K)$, and relate this group to the automorphism groups of its ellipsoids. We show that if $\operatorname{Aut}(K)$ is large enough, then the complexity of determining the ellipsoids $\mathrm{CE}(K)$ and $\operatorname{IE}(K)$ is greatly reduced, and in some cases, the ellipsoids can be determined explicitly. We then use this technique to compute the extremal ellipsoids associated with some classes of convex bodies that have important applications in convex optimization, namely when the convex body $K$ is the part of a given ellipsoid between two parallel hyperplanes, and when $K$ is a truncated second-order cone or an ellipsoidal cylinder.

Keywords: circumscribed ellipsoid, inscribed ellipsoid, John ellipsoid, Löwner ellipsoid, minimum-volume ellipsoid, maximum-volume ellipsoid, optimality conditions, semi-infinite programming, contact points, automorphism group, symmetric convex bodies, Haar measure

Subject classification: primary: 90C34, 46B20, 90C30, 90C46, 65K10; secondary: 52A $38,52 \mathrm{~A} 20,52 \mathrm{~A} 21,22 \mathrm{C} 05,54 \mathrm{H} 15$
\end{abstract}

\footnotetext{
* Department of Mathematics and Statistics, University of Maryland Baltimore County, Baltimore, Maryland 21250, USA. E-mail: guler@umbc.edu,gurtuna@magma-da.com. Research partially supported by the National Science Foundation under grant DMS-0411955
} 


\section{Introduction}

A convex body in $\mathbb{R}^{n}$ is a compact convex set with nonempty interior. Let $K$ be a convex body in $\mathbb{R}^{n}$. Among the ellipsoids circumscribing $K$, there exists a unique one with minimum volume and similarly, among the ellipsoids inscribed in $K$, there exists a unique one of maximum volume. These are called the minimal circumscribed ellipsoid and maximal inscribed ellipsoid of $K$, and we denote them by $\mathrm{CE}(K)$ and $\operatorname{IE}(K)$, respectively. To our knowledge, Behrend [6] is the first person to investigate these problems, and proves the existence and uniqueness of the two ellipsoids in the plane, that is when $n=2$. In any dimension, the existence of either ellipsoid is easy to prove using compactness. The ellipsoid $\mathrm{CE}(K)$ is often referred to as Löwner ellipsoid since Löwner has used its uniqueness in his lectures, see [10. The uniqueness of $\mathrm{CE}(K)$ also follows from the famous paper of John [18] although he does not state it explicitly. Subsequently, Danzer, Laugwitz, and Lenz [11, and Zaguskin [39] prove the uniqueness of both ellipsoids. The inscribed ellipsoid problem $\mathrm{IE}(K)$ is often called John ellipsoid, and sometimes Löwner-John ellipsoid, especially in Banach space geometry literature. (This terminology may seem inappropriate, since John does not consider the problem $\operatorname{IE}(K)$ in [18. However, in Banach space geometry literature, one is mainly interested in symmetric convex bodies, that is $K=-K$, and for this class of convex bodies the inscribed and circumscribed ellipsoids are related by polarity, so that results about one ellipsoid may be translated into a similar statement about the other.)

The two extremal problems $\mathrm{CE}(K)$ and $\operatorname{IE}(K)$ are important in several fields including optimization, Banach space geometry, and statistics. They also have applications in differential geometry 23, Lie group theory [11, and symplectic geometry, among others. The ellipsoid algorithm of Khachiyan [19] for linear programming sparked general interest of optimizers in the circumscribed ellipsoid problem. At the $k$ th step of this algorithm, one has an ellipsoid $E^{(k)}$ and is interested in finding $E^{(k+1)}=\mathrm{CE}(K)$ where $K$ is the intersection of $E^{(k)}$ with a half plane whose bounding hyperplane passes through the center of $E^{(k)}$. The ellipsoid $E^{(k+1)}$ can be computed explicitly, and the ratio of the volumes $\operatorname{vol}\left(E^{(k+1)}\right) / \operatorname{vol}\left(E^{(k)}\right)$ determines the rate of convergence of the algorithm and gives its polynomial-time complexity. From this perspective, ellipsoids covering the intersection of an ellipsoid with two halfspaces whose bounding hyperplanes are parallel have been studied as well, because the resulting ellipsoid algorithms are likely to have faster convergence. See the survey paper [7] and the book [13] a wealth of information on the of the ellipsoid method and its applications in optimization. The papers of König and Pallaschke 22 and Todd [36 compute the circumscribing ellipsoid explicitly. Inscribed ellipsoid problems also arise in optimization. For example, the inscribed ellipsoid method of Tarasov, Khachiyan, and Erlikh 34 is a polynomial-time algorithm for solving general convex optimization problems. In this method, one needs to compute numerically an approximation to the inscribed ellipsoid of a polytope which is described by a set of linear inequalities. A related paper of Khachiyan and Todd 20] gives a polynomial bound on the complexity of approximating the maximal inscribed ellipsoid for a polytope.

It has been discovered that the circumscribed ellipsoid problem is (dually) related to the optimal design problem in statistics. Consequently, there has been wide interest in the problem $\mathrm{CE}(K)$ and related problems in this community, see for example Wynn 37 . and Sibson's discussion following this paper, Fedorov [12, Titterington [35, Gruber [14, Ahipaşaoğlu [1, among others. The recent paper [17 by the second author gives a careful treatment of the duality theory of extremal ellipsoids and their connection to optimal design, using convex duality theory in a functional analysis setting. 
To meet the demand from different fields such as optimization, computer science, engineering, and statistics, there have been many algorithms proposed to numerically compute the two extremal ellipsoids $\mathrm{CE}(K)$ and $\operatorname{IE}(K)$. Recently, there has been a surge of research activity in this subject. We do not discuss algorithms in this paper, but the interested reader can find more information on this topic and the relevant references in the papers [40, 33, [2], 38], and [1].

This paper has the following, related goals. First, we investigate the symmetry properties of a convex body in $K \subset \mathbb{R}^{n}$ by studying $\operatorname{Aut}(K)$, the group of affine transformations leaving $K$ invariant. We then use the connection between of the automorphism group of $K$ and the automorphism groups of the extremal ellipsoids $\mathrm{CE}(K)$ and $\operatorname{IE}(K)$ to reduce of computational complexity of determining the two ellipsoids. Finally, we completely determine the ellipsoids for some classes of convex bodies, namely when the convex body $K$ is the part of a given ellipsoid between two parallel hyperplanes, and when $K$ is a truncated second-order cone or an ellipsoidal cylinder.

In $\$ 2$, we introduce semi-infinite programming formulations of the problems $\mathrm{CE}(K)$ and $\mathrm{IE}(K)$, and then recall their fundamental properties using the resulting optimality conditions. We devote $\$ 3$ to the symmetry properties of convex bodies and the related symmetry properties of the corresponding ellipsoids $\mathrm{CE}(K)$ and $\mathrm{IE}(K)$. One way to formalize the symmetry properties of a convex body $K$ is to consider its (affine) automorphism group $\operatorname{Aut}(K)$. It will be seen that the uniqueness of the two ellipsoids imply that the ellipsoids inherit the symmetry properties of the underlying convex body $K$. That is, $\operatorname{Aut}(K)$ is contained in the automorphism group of the two extremal ellipsoids. One consequence of this is that if $K$ is "symmetric" enough, then either it is possible to analytically compute the extremal ellipsoids exactly, or else it is possible to reduce the complexity of their numerical computation.

The contents of the rest of the paper are as follows. Starting with 4 , we exploit automorphism groups to analytically compute the extremal ellipsoids for two classes of convex bodies. The first class consists of convex bodies which are the intersections of a given ellipsoid with two halfspaces whose bounding hyperplanes are parallel, and have been mentioned above. We call such convex bodies slabs. The second class consists of convex bodies obtained by slicing an ellipsoid with two parallel hyperplanes and taking the convex hull of the slices. We note that a convex body in this class is either a truncated second-order cone or an ellipsoidal cylinder, depending on the location of the bounding hyperplanes with respect to the center of the ellipsoid.

In $\$ 4$, we compute the automorphism group of a slab $K$ and use it to determine the form of the center and matrix of its ellipsoid $\mathrm{CE}(K)$. Although the automorphism group $\operatorname{Aut}(K)$ is not large enough to compute the ellipsoid $\mathrm{CE}(K)$ exactly, it is large enough to reduce its determination to computing just three parameters (instead of $n(n+3) / 2$ in the general case), one to determine its center and two to determine its matrix.

In $\$ 5$, we formulate the $\mathrm{CE}(K)$ problem for a slab as a semi-infinite optimization problem, and obtain its solution by computing the three parameters of $\mathrm{CE}(K)$ directly from the Fritz John optimality conditions for the semi-infinite program. As we mentioned already, König and Pallaschke [22] and Todd [36] solve this exact problem. König and Pallaschke's approach is similar to ours: they use the uniqueness and invariance properties of the ellipsoid $\mathrm{CE}(K)$. However, their solution is not complete since they only consider the cases when the slab does not contain the center of the given ellipsoid. Todd gives a complete proof covering all cases. His proof is based on guessing the optimal ellipsoid and then prov- 
ing its minimality by using some bounds on the volume of a covering ellipsoid. In $\$ 5$, we also formulate the ellipsoid problem $\mathrm{CE}(K)$ as a nonlinear programming problem, and give a second, independent, solution for it.

For interesting applications of the ellipsoid $\mathrm{CE}(K)$ of a slab, see the papers [25] and [4].

In $\$ 6$, we formulate the $\operatorname{IE}(K)$ problem for a slab as a semi-infinite optimization problem, and obtain its solution directly from the resulting Fritz John optimality conditions. We also formulate the same problem as a nonlinear programming problem, but do not provide its solution in order to keep the length of the paper within reasonable bounds.

Finally, in $\$ 7$ we formulate the $\mathrm{CE}(K)$ problem for a convex body from the second class of convex bodies mentioned above as a semi-infinite programming problem, and obtain its solution directly from Fritz John optimality conditions. The form of the optimal ellipsoid $\mathrm{CE}(K)$ turns out to be very similar to the form of the corresponding ellipsoid for a slab. We do not solve the inscribed ellipsoid problem for the second class of convex bodies for space considerations.

We remark that the ideas and techniques used in this paper for determining the extremal ellipsoids for specific classes of convex bodies can be generalized to other classes of convex bodies as long as these bodies have large enough automorphism groups. It is reasonable to expect that automorphism groups can also be used advantageously in numerical determination of extremal ellipsoids.

Our notation is fairly standard. We denote the set of symmetric $n \times n$ matrices by $\mathbb{S R}^{n \times n}$. In $\mathbb{R}^{n}$, we use the bracket notation for inner products, thus $\langle u, v\rangle=u^{T} v$. In the vector space $\mathbb{R}^{n \times n}$ of $n \times n$ matrices (and hence in $\mathbb{S R}^{n \times n}$ ), we use the trace inner product

$$
\langle X, Y\rangle=\operatorname{tr}\left(X Y^{T}\right) .
$$

If both inner products are used within the same equation, then the meaning of each inner product should be clear from the context. We define and use additional inner products in this paper, especially in $\$ 3$ The sets $\partial X$ and $\operatorname{conv}(X)$ denote the boundary and the convex hull of a set $X$ in $\mathbb{R}^{n}$, respectively, and $\operatorname{ext}(K)$ is the set of extreme points of a convex set $K$ in $\mathbb{R}^{n}$.

\section{Characterizations of the extremal ellipsoids}

We recall that the circumscribed ellipsoid problem is the problem of finding a minimum volume ellipsoid circumscribing a convex body $K$ in $\mathbb{R}^{n}$. This is the main problem treated in Fritz John [18. In this paper, John shows that such an ellipsoid exists and is unique. John introduces semi-infinite programming and develops his optimality conditions to prove the following deep result about the ellipsoid $\mathrm{CE}(K)$ : the ellipsoid with the same center as $\mathrm{CE}(K)$ but shrunk by a factor $n$ is contained in $K$, and if $K$ is symmetric $(K=-K)$, then $\mathrm{CE}(K)$ needs to be shrunk by a smaller factor, which is $\sqrt{n}$, to be contained in $K$. This fact is very important in the geometric theory of Banach spaces. In that theory, a symmetric convex body $K$ is the unit ball of a Banach space, and if $K$ is an ellipsoid, then the Banach space is a Hilbert space. Consequently, the shrinkage factor indicates how close (in the Banach-Mazur distance) the Banach space is to being a Hilbert space; see [27, Chapter 3. In this context, it is not important to compute the exact ellipsoid $\mathrm{CE}(K)$. However, in some convex programming algorithms, including the ellipsoid method and its variants, the 
exact or nearly exact ellipsoid $\mathrm{CE}(K)$ needs to be computed. If $K$ is sufficiently simple, $\mathrm{CE}(K)$ can be computed analytically. In more general cases, numerical algorithms have been developed to approximately compute $\mathrm{CE}(K)$.

An ellipsoid $E$ in $\mathbb{R}^{n}$ is an affine image of the unit ball $B_{n}:=\left\{u \in \mathbb{R}^{n}:\|u\| \leq 1\right\}$, that is,

$$
E=c+A\left(B_{n}\right)=\left\{c+A u: u \in \mathbb{R}^{n},\|u\|=1\right\} \subset \mathbb{R}^{m},
$$

where $A \in \mathbb{R}^{m \times n}$ is any $m \times n$ matrix. Here $c$ is the center of $E$ and the volume of $E$ is given by $\operatorname{vol}(E)=\operatorname{det}(A) \operatorname{vol}\left(B_{n}\right)$. We are interested in the case where $E$ is a solid body ( $E$ has a non-empty interior), hence we assume that $A$ is a non-singular $n \times n$ matrix. Let $A$ have the singular value decomposition $A=V_{1} \Sigma V_{2}$ where $V_{1}, V_{2}$ are orthogonal $n \times n$ matrices and $\Sigma$ is a diagonal matrix with positive elements. Then we have the polar decomposition of $A$, that is, $A=S O$ where $S=V_{1} A V_{1}^{T} \in \mathbb{S R}^{n \times n}$ is positive definite and $O=V_{1} V_{2}$ is an orthogonal matrix. Consequently, $E=c+S O\left(B_{n}\right)=c+S\left(B_{n}\right)$, that is, the matrix $A$ in the definition of the ellipsoid $E$ in (2.1) can be taken to be symmetric and positive definite, an assumption we make from here on. By making the change of variables $x:=c+A u$, that is, $u=A^{-1}(x-c)$, and defining $X:=A^{-2}$, the ellipsoid $E$ in (2.1), $E=\left\{x \in \mathbb{R}^{n}:\left\|A^{-1}(x-c)\right\|^{2} \leq 1\right\}$ can also be written in the form

$$
E=E(X, c):=\left\{x \in \mathbb{R}^{n}:\langle X(x-c), x-c\rangle \leq 1\right\} .
$$

Note that we have

$$
\operatorname{vol}(E)=\operatorname{det}(X)^{-1 / 2} \omega_{n}
$$

where $\omega_{n}=\operatorname{vol}\left(B_{n}\right)$.

Consequently, we can set up the circumscribed ellipsoid problem as a semi-infinite program

$$
\begin{array}{ll}
\min & -\log \operatorname{det} X \\
\text { s.t. } & \langle X(y-c), y-c\rangle \leq 1, \quad \forall y \in K,
\end{array}
$$

in which the decision variables are $X \in \mathbb{S R}^{n \times n}$ and $c \in \mathbb{R}^{n}$.

The following fundamental theorem of Fritz John [18 is a basic tool in semi-infinite programming. It is proved, for example, in [16], Chapter 12.

Theorem 2.1. (Fritz John) Consider the optimization problem

$$
\begin{array}{cl}
\min & f(x) \\
\text { s.t. } & g(x, y) \leq 0, \quad \forall y \in Y,
\end{array}
$$

where $f(x)$ is a continuously differentiable function defined on an open set $X \subseteq \mathbb{R}^{n}$, and $g(x, y)$ and $\nabla_{x} g(x, y)$ are continuous functions defined on $X \times Y$ where $Y$ is a compact set in some topological space. If $x$ is a local minimizer of (2.5), then there exist at most $n$ active constraints $\left\{g\left(x, y_{i}\right)\right\}_{1}^{k}\left(g\left(x, y_{i}\right)=0\right)$ and a non-trivial, non-negative multiplier vector $0 \neq\left(\lambda_{0}, \lambda_{1}, \ldots, \lambda_{k}\right) \geq 0$ such that

$$
\lambda_{0} \nabla f(x)+\sum_{i=1}^{k} \lambda_{i} \nabla_{x} g\left(x, y_{i}\right)=0 .
$$

The following theorem characterizes the circumscribed ellipsoid $\mathrm{CE}(K)$. It can be proved by applying Theorem 2.1 to the semi-infinite program [2.4], see [16], section 12.3. 
Theorem 2.2. If $K$ is convex body in $\mathbb{R}^{n}$, then there exists a unique ellipsoid $\mathrm{CE}(K)$ of minimum volume circumscribing $K$. Moreover, an ellipsoid $E(X, c)$ is the ellipsoid $\mathrm{CE}(K)$ if and only if there exists a multiplier vector $\lambda=\left(\lambda_{1}, \ldots, \lambda_{k}\right)>0,0 \leq k \leq n(n+3) / 2$, and points $\left\{u_{i}\right\}_{1}^{k}$ in $K$ such that

$$
\begin{aligned}
X^{-1} & =\sum_{i=1}^{k} \lambda_{i}\left(u_{i}-c\right)\left(u_{i}-c\right)^{T}, \\
0 & =\sum_{i=1}^{k} \lambda_{i}\left(u_{i}-c\right), \\
u_{i} & \in \partial K \cap \partial E(X, c), \quad i=1, \ldots, k, \\
K & \subseteq E(X, c) .
\end{aligned}
$$

The first equation above gives $I=\sum_{i=1}^{k} \lambda_{i} X\left(u_{i}-c\right)\left(u_{i}-c\right)^{T}$, and taking traces of both sides yields $n=\operatorname{tr}\left(I_{n}\right)=\operatorname{tr}\left(\sum_{i=1}^{k} \lambda_{i} X u_{i} u_{i}^{T}\right)=\sum_{i=1}^{k} \lambda_{i}\left\langle X u_{i}, u_{i}\right\rangle=\sum_{i=1}^{k} \lambda_{i}$, that is,

$$
\sum_{i=1}^{k} \lambda_{i}=n .
$$

We call the points $\left\{u_{i}\right\}_{1}^{k}$ in $\partial K \cap \partial E$ contact points of $K$ and $E$. The equation $\sum_{i=1}^{k} \lambda_{i}\left(u_{i}-c\right)=0$ in 2.6 gives $c \in \operatorname{conv}\left(\left\{u_{i}\right\}_{1}^{k}\right)$. This immediately implies

Corollary 2.3. Let $K$ be a convex body in $\mathbb{R}^{n}$. The contact points of $\mathrm{CE}(K)$ are not contained in any closed halfspace whose bounding hyperplane passes through the center of $\mathrm{CE}(K)$.

Remark 2.4. The contact points have applications in several fields, in optimal designs, and in estimating the size of almost orthogonal submatrices of orthogonal matrices [31, for example. Gruber [14] shows that "most" convex bodies $K$ have the maximum number $n(n+3) / 2$ of contact points. See 31 for a simpler proof. Similar results also hold for the maximum volume inscribed ellipsoids, see [14. However, Rudelson 31] shows that for every $\varepsilon>0$ and every convex body $K$, there exists a nearby convex body $L$ whose distance (Banach-Mazur distance) to $K$ is less than $1+\varepsilon$ and which at most $k \leq C(\varepsilon) \cdot n \log ^{3} n$ contact points. This has obvious implications for numerical algorithms that try to compute approximate covering ellipsoids.

Remark 2.5. Let $S=\operatorname{ext}(K)$, the set of extreme points of $K$. We have $K=\operatorname{conv}(S)$, the convex hull of $S$, by a Theorem of Minkowski, see Rockafellar [30, Corollary 18.5.1. Note that $S$ and $K$ have the same extremal covering ellipsoid, and applying Theorem 2.2 to $S$ instead of $K$ shows that we can choose $u_{i} \in S=\operatorname{ext}(K), i=1, \ldots, k$.

An independent proof of the above fact runs as follows: let $x \in \partial K \cap \partial E$ be a contact point. Noting $\partial E=\operatorname{ext}(E)$, we have $x \in \operatorname{ext}(E)$. If $x \notin \operatorname{ext}(K)$, then there exist $y, z \in K$, $y \neq z$, such that $x$ lies in the interior of the line segment $[y, z]$. However, $x \in[y, z] \subseteq E$, contradicting the fact that $x \in \operatorname{ext}(E)$.

The concept of the support function will be needed in the formulation of the inscribed ellipsoid problem. If $C$ is a convex body in $\mathbb{R}^{n}$, then the Minkowski support function of $C$ is defined by 


$$
s_{C}(d):=\max _{u \in C}\langle d, u\rangle
$$

The function $s_{C}$ is clearly defined on $\mathbb{R}^{n}$ and is a convex function because it is a maximum of linear functions indexed by $u$. If $C$ and $D$ are two convex bodies, it follows from Corollary 13.1.1 in [30] that $C \subseteq D$ if and only if $s_{C} \leq s_{D}$.

We compute

$$
\begin{aligned}
s_{E(X, c)}(d) & =\max \{\langle d, u\rangle:\langle X(u-c), u-c\rangle \leq 1\} \\
& \left.=\max \left\{\left\langle d, c+X^{-1 / 2} v\right\rangle:\|v\| \leq 1\right\rangle\right\} \\
& =\langle c, d\rangle+\max _{\|v\|=1}\left\langle X^{-1 / 2} d, v\right\rangle=\langle c, d\rangle+\left\|X^{-1 / 2} d\right\| \\
& =\langle c, d\rangle+\left\langle X^{-1} d, d\right\rangle^{1 / 2},
\end{aligned}
$$

where we have defined $v=X^{1 / 2}(u-c)$ or $u=c+X^{-1 / 2} v$.

We can formulate the inscribed ellipsoid problem as a semi-infinite program

$$
\min \{\operatorname{det} X: E(X, c) \subseteq K\} .
$$

However, this is hard to work with, due to the inconvenient form of the constraints, $E(X, c) \subseteq K$. We replace this inclusion by the functional constraints

$$
s_{E(X, c)}(d) \leq s_{K}(d), \quad \forall d \in B_{n},
$$

where we again restrict $d$ to the unit sphere since support functions are homogeneous (of degree 1), in order to get a compact indexing set.

Defining $Y=X^{-1}$, we can therefore rewrite our semi-infinite program in the form

$$
\begin{array}{ll}
\min & -\log \operatorname{det} Y \\
\text { s.t. } & \langle c, d\rangle+\langle Y d, d\rangle^{1 / 2} \leq s_{K}(d), \quad \forall d:\|d\|=1,
\end{array}
$$

in which the decision variables are $(Y, c) \in S^{n} \times \mathbb{R}^{n}$ and we have infinitely many constraints indexed by the unit vector $\|d\|=1$.

Since $s_{K}$ is a convex function on $\mathbb{R}^{n}$, it is continuous. Therefore, there exists a positive constant $M>0$ such that if $(Y, c)$ is a feasible decision variable, then $|\langle c, d\rangle| \leq M$, and $\langle Y d, d\rangle \leq M$ for all $\|d\|=1$. This proves that the set of feasible $(Y, c)$ for problem (2.9) is compact, and implies that there exists a maximum volume ellipsoid inscribed in $K$.

The theorem below characterizes the inscribed ellipsoid $\operatorname{IE}(K)$. It can be proved by applying Theorem 2.1 to the semi-infinite program (2.9), see [16], section 12.4.

Theorem 2.6. If $K$ is a convex body in $\mathbb{R}^{n}$, then there exists a unique ellipsoid $\operatorname{IE}(K)$ of maximum volume inscribed in $K$. If an ellipsoid $E(X, c)$ contained in $K$ is the ellipsoid $\operatorname{IE}(K)$ if and only if there exists a multiplier vector $\lambda=\left(\lambda_{1}, \ldots, \lambda_{k}\right)>0,0 \leq k \leq n(n+3) / 2$, and contact points $\left\{u_{i}\right\}_{1}^{k}$ such that

$$
\begin{aligned}
X^{-1} & =\sum_{i=1}^{k} \lambda_{i}\left(u_{i}-c\right)\left(u_{i}-c\right)^{T}, \\
0 & =\sum_{i=1}^{k} \lambda_{i}\left(u_{i}-c\right), \\
u_{i} & \in \partial K \cap \partial E(X, c), \quad i=1, \ldots, k, \\
E(X, c) & \subseteq K .
\end{aligned}
$$


We note that the optimality conditions 2.10 are exactly the same as the corresponding optimality conditions (2.6) in the circumscribed ellipsoid case, except for the feasibility conditions $E(X, c) \subseteq K$.

As in the circumscribed ellipsoid case, we have

Corollary 2.7. Let $K$ be a convex body in $\mathbb{R}^{n}$. The contact points of $\operatorname{IE}(K)$ are not contained in any closed halfspace whose bounding hyperplane passes through the center of $\operatorname{IE}(K)$.

Remark 2.8. The minimum volume covering ellipsoid problem may be set as a semi-infinite program in a different way, by replacing the set inclusion $K \subseteq E(X, c)$ by the equivalent functional constraints $s_{E(X, c)}(d) \geq s_{K}(d)$, that is by the constraints

$$
\langle c, d\rangle+\langle Y d, d\rangle^{1 / 2} \geq s_{K}(d), \quad \forall d,\|d\|=1,
$$

where we restrict $d$ to the unit sphere since support functions are homogeneous (of degree 1 ), in order to get a compact indexing set. The resulting semi-infinite program is solved in the same way as (2.4), and mirrors the solution to the maximum volume inscribed ellipsoid problem given above.

\section{Automorphism group of a convex body}

Let $K$ be a convex body in $\mathbb{R}^{n}$. The uniqueness of the two extremal ellipsoids $\mathrm{CE}(K)$ and $\mathrm{IE}(K)$ have important consequences regarding the invariance properties of the two ellipsoids. We will see in this section that the symmetry properties of the convex body $K$ is inherited by the two ellipsoids. If $K$ is symmetric enough, then it becomes possible to give explicit formulae for the ellipsoids $\operatorname{CE}(K)$ and $\operatorname{IE}(K)$. We will demonstrate this for some special convex bodies in the remaining sections of this paper.

Apart from its intrinsic importance, the invariance properties of the ellipsoids $\mathrm{CE}(K)$ and $\mathrm{IE}(K)$ have important applications to Lie groups [11], [26], to differential geometry [23], and to the computation of the extremal ellipsoids for some special polytopes and convex bodies [8], 5], among others.

We start with a definition.

Definition 3.1. The (affine) automorphism group $\operatorname{Aut}(K)$ of a convex body $K$ in $\mathbb{R}^{n}$ is the set of affine transformations $T(x)=a+A x$ leaving $K$ invariant, that is,

$$
\operatorname{Aut}(K):=\{T(x)=a+A x: T(K)=K\} .
$$

Note that since $0<\operatorname{vol}(K)=\operatorname{vol}(T(K))=|\operatorname{det} A| \operatorname{vol}(K)$, we have $|\operatorname{det} A|=1$.

It is shown in 14 that the automorphism group of most convex bodies consists of the identity transformation alone. This is to be expected, since the symmetry properties of a given convex body can easily be destroyed by slightly perturbing the body. Nevertheless, the study of the symmetry properties of convex bodies is important for many reasons.

The ellipsoids are the most symmetric convex bodies. Therefore, we first investigate their automorphism groups and then relate them to the automorphism groups of arbitrary convex bodies. 
Definition 3.2. Let $A$ be an invertible matrix in $\mathbb{S R}^{n \times n}$. Equip $\mathbb{R}^{n}$ with the quadratic form $\langle A u, v\rangle$ which we write as an inner product

$$
\langle u, v\rangle_{A}:=\langle A u, v\rangle .
$$

We denote by $\mathcal{O}\left(\mathbb{R}^{n}, A\right)$ the set of linear maps orthogonal under this inner product,

$$
\begin{aligned}
\mathcal{O}\left(\mathbb{R}^{n}, A\right) & :=\left\{g \in \mathbb{R}^{n \times n}: g^{*} g=g g^{*}=I\right\} \\
& =\left\{g \in \mathbb{R}^{n \times n}:\langle g u, g v\rangle_{A}=\langle u, v\rangle_{A}, \forall u, v \in \mathbb{R}^{n}\right\} \\
& =\left\{g \in \mathbb{R}^{n \times n}: g^{T} A g=A\right\},
\end{aligned}
$$

where $g^{*}$ is the conjugate matrix of $g$ with respect to the inner product $\langle\cdot, \cdot\rangle_{A}$, that is $\langle g u, v\rangle_{A}=\left\langle u, g^{*} v\right\rangle_{A}$ for all $u, v$ in $\mathbb{R}^{n}$. The second equality above follows as $\langle g u, g v\rangle_{A}=$ $\left\langle u, g^{*} g v\right\rangle_{A}$ and this equals $\langle u, v\rangle_{A}$ if and only if $g^{*} g=I$. The third equality follows since $\left\langle g^{T} A g u, v\right\rangle=\langle A g u, g v\rangle=\langle g u, g v\rangle_{A}=\langle u, v\rangle_{A}=\langle A u, v\rangle$. If $A$ is positive definite, then $\left(\mathbb{R}^{n},\langle\cdot, \cdot\rangle_{A}\right)$ is a Euclidean space. In particular, $\mathcal{O}_{n}:=\mathcal{O}\left(\mathbb{R}^{n}, I\right)$ is the set of orthogonal matrices in the usual inner product on $\mathbb{R}^{n}$.

Lemma 3.3. If $X$ is a symmetric, positive definite $n \times n$ matrix, then

$$
\operatorname{Aut}(E(X, 0))=\mathcal{O}\left(\mathbb{R}^{n}, X\right) .
$$

In particular, $\operatorname{Aut}\left(B_{n}\right)$ is the set of $n \times n$ orthogonal matrices. We also have

$$
\operatorname{Aut}(E(X, c))=T_{c} \mathcal{O}\left(\mathbb{R}^{n}, X\right) T_{-c}
$$

where $T_{c}$ is the translation map $T_{c} x=c+x$. Moreover, $\operatorname{Aut}(E(X, c))$ fixes $c$, the center of $E(X, c)$, that is, $\theta(c)=c$ for every $\theta$ in $\operatorname{Aut}(E(X, c))$.

Proof. We first determine $\operatorname{Aut}\left(B_{n}\right)$. Let $T$ be in $\operatorname{Aut}\left(B_{n}\right)$, where $T(x)=a+A x$. Since $T$ maps the boundary of $B_{n}$ onto itself, we have $q(x):=\|a+A x\|^{2}=\left\langle A^{T} A x, x\right\rangle+2\left\langle A^{T} a, x\right\rangle+$ $\|a\|^{2}=1$ for all $\|x\|=1$. Then $q(x)-q(-x)=4\left\langle A^{T} a, x\right\rangle=0$ for all $\|x\|=1$, which implies that $A^{T} a=0$ and since $A$ is invertible, $a=0$. Consequently, $q(x)=\left\langle A^{T} A x, x\right\rangle=1$ for all $\|x\|=1$, which gives $A^{T} A=I_{n}$, that is, $A$ is an orthogonal matrix.

Next, we determine $\operatorname{Aut}(E(X, 0))$. We have the commutative diagram

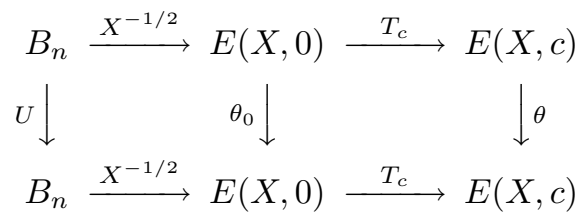

where $\theta \in \operatorname{Aut}(E(X, c)), U \in \operatorname{Aut}\left(B_{n}\right)=\mathcal{O}_{n}$, and $\theta_{0} \in \operatorname{Aut}(E(X, 0))$. From the diagram, we have $I=U^{T} U=\left(X^{1 / 2} \theta_{0} X^{-1 / 2}\right)^{T}\left(X^{1 / 2} \theta_{0} X^{-1 / 2}\right)=X^{-1 / 2} \theta_{0}^{T} X \theta_{0} X^{-1 / 2}$. This gives $\theta_{0}^{T} X \theta_{0}=X$ and proves that $\operatorname{Aut}(E(X, 0))=\mathcal{O}\left(\mathbb{R}^{n}, X\right)$.

Since $T_{c}^{-1}=T_{-c}$, we have

$$
\operatorname{Aut}(E(X, c))=T_{c} \circ \operatorname{Aut}(E(X, 0)) \circ T_{-c} .
$$

Every $\theta$ in $\operatorname{Aut}(E(X, c))$ has the form $\theta(u)=\left(T_{c} \circ \theta_{0} \circ T_{-c}\right)(u)=T_{c}\left(\theta_{0}(-c+u)\right)=$ $\left(c-\theta_{0} c\right)+\theta_{0} u$ for some $\theta_{0}$ in $\operatorname{Aut}\left(E_{0}\right)$. This gives $\theta(c)=c$, meaning that $\operatorname{Aut}(E(X, c))$ fixes the center of $E$. 
Definition 3.4. Let $K$ be a convex body in $\mathbb{R}^{n}$. An ellipsoid $E=E(X, c)$ is an invariant ellipsoid of $K$ if $\operatorname{Aut}(K) \subseteq \operatorname{Aut}(E)$, that is, if every automorphism of $K$ is an automorphism of $E$.

It immediately follows from Lemma 3.3 that $\operatorname{Aut}(K)$ fixes the center of any invariant ellipsoid $E$ of $K$.

The following Theorem in Danzer et al. [11 is a central result regarding the symmetry properties of the extremal ellipsoids.

Theorem 3.5. Let $K$ be a convex body in $\mathbb{R}^{n}$. The extremal ellipsoids $\mathrm{CE}(K)$ and $\operatorname{IE}(K)$ are invariant ellipsoids of $K$. Thus, $\operatorname{Aut}(K) \subseteq \operatorname{Aut}(\mathrm{CE}(K)), \operatorname{Aut}(K) \subseteq \operatorname{Aut}(\operatorname{IE}(K))$, and $\operatorname{Aut}(K)$ fixes the centers of $\mathrm{CE}(K)$ and $\operatorname{IE}(K)$.

Proof. Since the arguments are similar, we only prove the statements about $\mathrm{CE}(K)$. Let $g \in$ Aut $(K)$. Since $K \subseteq \mathrm{CE}(K)$ and $K=g K \subseteq g(\mathrm{CE}(K))$, the ellipsoids $\mathrm{CE}(K)$ and $g(\mathrm{CE}(K))$ both cover $K$, and since $\operatorname{vol}(\mathrm{CE}(K))=\operatorname{vol}(g(\mathrm{CE}(K)))$, they are both minimum volume circumscribed ellipsoids of $K$. It follows from Theorem 2.2 that $g(\mathrm{CE}(K))=\mathrm{CE}(K)$.

Corollary 3.6. The automorphism group $\operatorname{Aut}(K)$ of a convex body $K$ in $\mathbb{R}^{n}$ is a compact Lie group.

Proof. We have $\operatorname{Aut}(K) \subseteq \operatorname{Aut}(\mathrm{CE}(K))$ by Theorem 3.5 and Lemma 3.3 implies that $\operatorname{Aut}(\mathrm{CE}(K))$ is compact. Clearly, $\operatorname{Aut}(K)$ is a closed subset of the general affine linear group in $\mathbb{R}^{n}$. A classical theorem of von Neumann implies that it is a Lie group; see Knapp 21] for a proof (Theorem 0.15, pp. 11-15) and historical notes (p. 753). Clearly, Aut(K) is a compact group.

\section{Automorphism group of a slab}

In this paper, one of the problems we are interested in is the determination of the extremal ellipsoids of the convex body $K$ which is the part of a given ellipsoid $E\left(X_{0}, c_{0}\right)$ between two parallel hyperplanes,

$$
K=\left\{x:\left\langle X_{0}\left(x-c_{0}\right), x-c_{0}\right\rangle \leq 1, a \leq\left\langle p, x-c_{0}\right\rangle \leq b\right\},
$$

where $p$ is a non-zero vector in $\mathbb{R}^{n}$, and where $a$ and $b$ are such that $K$ is nonempty. Recall that we call such a convex body $K$ a slab.

In this section, we determine $\operatorname{Aut}(K)$ and the form of the ellipsoids $\operatorname{CE}(K)$ and $\operatorname{IE}(K)$.

If we substitute $u=X_{0}^{1 / 2}\left(x-c_{0}\right)$, that is, $x=c_{0}+X_{0}^{-1 / 2} u$, the quadratic inequality $\left\langle X_{0}\left(x-c_{0}\right), x-c_{0}\right\rangle \leq 1$ becomes $\|u\| \leq 1$ and making the further substitution $q:=X_{0}^{-1 / 2} p$, the linear form $\left\langle p, x-c_{0}\right\rangle$ becomes

$$
\left\langle p, x-c_{0}\right\rangle=\left\langle X_{0}^{-1 / 2} p, X_{0}^{1 / 2}\left(x-c_{0}\right)\right\rangle=\langle q, u\rangle .
$$

Defining $\bar{p}=q /\|q\|, \alpha=a /\|q\|$ and $\beta=b /\|q\|$, the linear inequalities $a \leq\left\langle p, x-c_{0}\right\rangle \leq b$ reduce to $\alpha \leq\langle\bar{p}, u\rangle \leq \beta$. Altogether, these substitutions give

$$
K=\left\{c_{0}+X_{0}^{-1 / 2} u:\|u\| \leq 1, \alpha \leq\langle\bar{p}, u\rangle \leq \beta\right\} .
$$


Let $Q$ be an orthogonal matrix such that $Q e_{1}=\bar{p}$, where $e_{1}=(1,0, \ldots, 0)^{T}$ in $\mathbb{R}^{n}$. Defining $v=Q^{-1} u$, we finally have

$$
K=\left\{c_{0}+X_{0}^{-1 / 2} Q v:\|v\| \leq 1, \alpha \leq\left\langle e_{1}, v\right\rangle \leq \beta\right\},
$$

that is, $K=c_{0}+X_{0}^{-1 / 2} Q(\tilde{K})$, where $\tilde{K}=\left\{v:\|v\| \leq 1, \alpha \leq\left\langle e_{1}, v\right\rangle \leq \beta\right\}$.

Since an affine transformation leaves ratios of volumes unchanged, we assume from here on, without loss of generality, that our initial convex body $K$, which we denote by $B_{\alpha \beta}$, has the form

$$
B_{\alpha \beta}=\left\{x \in \mathbb{R}^{n}:\|x\| \leq 1, \alpha \leq x_{1} \leq \beta\right\},
$$

where $-1 \leq \alpha<\beta \leq 1$.

Remark 4.1. To simplify our proofs, we assume in this paper that $\beta^{2} \geq \alpha^{2}$. We can always achieve this by working with the convex body $-B_{\alpha \beta}$ instead of $B_{\alpha \beta}$, if necessary.

We use the symmetry properties of $B_{\alpha \beta}$ to determine the possible forms of its extremal ellipsoids. This idea seems to be first suggested in 22 for determining $\operatorname{CE}\left(B_{\alpha \beta}\right)$.

Lemma 4.2. If $\alpha=-1$ and $\beta=1$, then the automorphism group of the slab $B_{\alpha \beta}=B_{n}$ consists of the $n \times n$ orthogonal matrices. In the remaining cases, the automorphism group $\operatorname{Aut}\left(B_{\alpha \beta}\right)$ consists of linear transformations $T(u)=A u$ where $A$ is a matrix of the form

$$
A=\left[\begin{array}{cc}
a_{11} & 0 \\
0 & \bar{A}
\end{array}\right], \quad a_{11} \in \mathbb{R}, \bar{A} \in \mathcal{O}_{n-1},
$$

with $a_{11}=1$ if $\alpha \neq-\beta$ and $a_{11}= \pm 1$ if $\alpha=-\beta$.

Proof. It is proved in Lemma 3.3 that $\operatorname{Aut}\left(B_{n}\right)=\mathcal{O}_{n}$, so we consider the remaining cases.

Let $T(u)=a+A u$ be an automorphism of $B_{\alpha \beta}$. We write $A=\left[\begin{array}{cc}a_{11} & c^{T} \\ b & \bar{A}\end{array}\right]$ and $a=\left(a_{1}, \bar{a}\right)$, where $a_{11}$ and $a_{1}$ are scalars and the rest of the variables have the appropriate dimensions. Since $T$ is an invertible affine map, $T\left(\operatorname{ext}\left(B_{\alpha \beta}\right)\right)=\operatorname{ext}\left(B_{\alpha \beta}\right)$, where $\operatorname{ext}\left(B_{\alpha \beta}\right)$ is the set of extreme points of $B_{\alpha \beta}$ given by

$$
\operatorname{ext}\left(B_{\alpha \beta}\right)=\left\{u=\left(u_{1},\left(1-u_{1}^{2}\right)^{1 / 2} \bar{u}\right): u_{1} \in[\alpha, \beta],\|u\|=1,\|\bar{u}\|=1\right\} .
$$

If $u=\left(u_{1},\left(1-u_{1}^{2}\right)^{1 / 2} \bar{u}\right)$ is $\operatorname{in} \operatorname{ext}\left(B_{\alpha \beta}\right)$ with $\|\bar{u}\|=1$, then

$$
w:=a+A u=\left[\begin{array}{c}
a_{11} u_{1}+\left(1-u_{1}^{2}\right)^{1 / 2}\langle c, \bar{u}\rangle+a_{1} \\
u_{1} b+\left(1-u_{1}^{2}\right)^{1 / 2} \bar{A} \bar{u}+\bar{a}
\end{array}\right] .
$$

We have $\|w\|=1$, that is,

$$
\begin{aligned}
1= & \left(a_{11} u_{1}+a_{1}\right)^{2}+\left(1-u_{1}^{2}\right)\langle c, \bar{u}\rangle^{2}+2\left(1-u_{1}^{2}\right)^{1 / 2}\langle c, \bar{u}\rangle\left(a_{11} u_{1}+a_{1}\right) \\
& +\left\|u_{1} b+\bar{a}\right\|^{2}+\left(1-u_{1}^{2}\right)\|\bar{A} \bar{u}\|^{2}+2\left(1-u_{1}^{2}\right)^{1 / 2}\left\langle\bar{A} \bar{u}, u_{1} b+\bar{a}\right\rangle \\
= & \left\{\left(a_{11} u_{1}+a_{1}\right)^{2}+\left\|u_{1} b+\bar{a}\right\|^{2}\right\}+\left(1-u_{1}^{2}\right)\left\langle\left(\bar{A}^{T} \bar{A}+c c^{T}\right) \bar{u}, \bar{u}\right\rangle \\
& +2\left(1-u_{1}^{2}\right)^{1 / 2}\left\langle\left(a_{11} u_{1}+a_{1}\right) c+\bar{A}^{T}\left(u_{1} b+\bar{a}\right), \bar{u}\right\rangle \\
= & : q(\bar{u}), \quad \forall\|\bar{u}\|=1 .
\end{aligned}
$$


Fix $u_{1} \in(\alpha, \beta)$, so that $1-u_{1}^{2} \neq 0$. The argument used in the proof of Lemma 3.3 shows that

$$
\left(a_{11} u_{1}+a_{1}\right) c+\bar{A}^{T}\left(u_{1} b+\bar{a}\right)=0, \quad \forall u_{1} \in(\alpha, \beta),
$$

and that $\left(1-u_{1}\right)^{2}\left\langle\left(\bar{A}^{T} \bar{A}+c c^{T}\right) \bar{u}, \bar{u}\right\rangle=\left\{1-\left(a_{11} u_{1}+a_{1}\right)^{2}-\left\|u_{1} b+\bar{a}\right\|^{2}\right\}\|\bar{u}\|^{2}$ for all $\bar{u} \in$ $\mathbb{R}^{n-1}$, that is,

$$
\left(1-u_{1}\right)^{2}\left(\bar{A}^{T} \bar{A}+c c^{T}\right)=\left\{1-\left(a_{11} u_{1}+a_{1}\right)^{2}-\left\|u_{1} b+\bar{a}\right\|^{2}\right\} I_{n-1},
$$

for all $u_{1} \in(\alpha, \beta)$. Therefore, there exists a constant $k$ such that

$$
\begin{aligned}
k I_{n-1} & =\bar{A}^{T} \bar{A}+c c^{T}, \\
0 & =\left(a_{11} u_{1}+a_{1}\right)^{2}+\left\|u_{1} b+\bar{a}\right\|^{2}+k\left(1-u_{1}^{2}\right)-1, \quad \forall u_{1} \in(\alpha, \beta) .
\end{aligned}
$$

The equation 4.3 implies the first two equations in 4.4 , while the equation above gives rest of the equations below,

$$
\begin{aligned}
& 0=\bar{A}^{T} b+a_{11} c, \quad 0=\bar{A}^{T} \bar{a}+a_{1} c, \quad k I_{n-1}=\bar{A}^{T} \bar{A}+c c^{T}, \\
& 0=a_{11}^{2}+\|b\|^{2}-k, \quad 0=a_{1} a_{11}+\langle b, \bar{a}\rangle, \quad 0=a_{1}^{2}+\|\bar{a}\|^{2}+k-1 .
\end{aligned}
$$

We have, therefore,

$$
A^{T} A=\left[\begin{array}{cc}
a_{11}^{2}+\|b\|^{2} & a_{11} c^{T}+b^{T} \bar{A} \\
a_{11} c+\bar{A}^{T} b & \bar{A}^{T} \bar{A}+c c^{T}
\end{array}\right]=\left[\begin{array}{cc}
k & 0 \\
0 & k I_{n-1}
\end{array}\right]=k I_{n} .
$$

Since $|\operatorname{det} A|=1$ and $A^{T} A$ is positive semidefinite, we have $k=1$. This proves that $A$ is an orthogonal matrix. Furthermore, the last equation in (4.5) gives $a=\left(a_{1}, \bar{a}\right)=0$.

Let $x$ be in $B_{\alpha \beta}$. As $\|A x\|=\|x\| \leq 1$ and $\left\langle e_{1}, x\right\rangle=\left\langle A e_{1}, A x\right\rangle$, we have

$$
\begin{aligned}
B_{\alpha \beta}=A B_{\alpha \beta} & =\left\{A x:\|x\| \leq 1, \alpha \leq\left\langle e_{1}, x\right\rangle \leq \beta\right\} \\
& =\left\{x:\|x\| \leq 1, \alpha \leq\left\langle A e_{1}, x\right\rangle \leq \beta\right\} .
\end{aligned}
$$

If $\alpha \neq-\beta$, then we must have $A e_{1}=e_{1}$, and if $\alpha=-\beta$, then $B_{\alpha \beta}=-B_{\alpha \beta}$ and we have $A e_{1}= \pm e_{1}$. Since $A e_{1}=\left[\begin{array}{c}a_{11} \\ b\end{array}\right]$, we see that $\left|a_{11}\right|=1$ and $b=c=0$. It is then clear that $\bar{A}$ belongs to $\mathcal{O}_{n-1}$.

Conversely, it is easy to verify that any matrix $A$ in the form above is in $\operatorname{Aut}(K)$.

\section{Determination of the circumscribed ellipsoid of a slab}

In this section, we give explicit formulae for the minimum volume circumscribed ellipsoid of the slab $B_{\alpha \beta}$ in (4.1) using the Fritz John optimality conditions 2.6 and Lemma 5.1 below. In this section, $K$ will always denote the convex body $B_{\alpha \beta}$.

Lemma 5.1. The extremal ellipsoids $\operatorname{CE}\left(B_{\alpha \beta}\right)$ and $\operatorname{IE}\left(B_{\alpha \beta}\right)$ have the form $E(X, c)$ where $c=\tau e_{1}$ and $X=\operatorname{diag}(a, b, \ldots, b)$ for some $a>0, b>0$ and $\tau$ in $\mathbb{R}$. Moreover, if $\alpha=-\beta$, then $c=0$. 
Proof. Since the proofs are the same, we consider only the covering ellipsoid $\operatorname{CE}\left(B_{\alpha \beta}\right)=$ $E(X, c)$. It follows from Lemma 3.3 that any $U=\left[\begin{array}{ll}1 & 0 \\ 0 & \bar{U}\end{array}\right]$ with $\bar{U} \in \mathcal{O}_{n-1}$ lies in $\operatorname{Aut}(K)$. Write $c=\left(c_{1}, \bar{c}\right)$. It follows from Theorem 3.5 that $U c=c$, and this implies that $\bar{U} \bar{c}=\bar{c}$ for all $\bar{U}$ in $\mathcal{O}_{n-1}$. Choosing $\bar{U}=-I_{n-1}$, we obtain $\bar{c}=0$. Moreover, if $\alpha=-\beta$, then $U=-I_{n}$ lies in $\operatorname{Aut}(K)$. Again, it follows from Theorem 3.5 that $U c=c$, and this yields $c=0$.

Next, Theorem 3.5 implies that $U=\left[\begin{array}{ll}1 & 0 \\ 0 & \bar{U}\end{array}\right] \in \operatorname{Aut}(K)$ above lies in $\operatorname{Aut}(E(X, c))$, and Lemma 3.3 implies that $U=T_{c} \circ \hat{U} \circ T_{-c}$ for some $\hat{U} \in \operatorname{Aut}(E(X, 0))=\mathcal{O}\left(\mathbb{R}^{n}, X\right)$. Since $U c=c$, we have $\hat{U} u=\left(T_{-c} \circ U \circ T_{c}\right)(u)=T_{-c}(U u+U c)=T_{-c}(U u+c)=U u$, that is, $\hat{U}=U$. It follows that $U \in \mathcal{O}\left(\mathbb{R}^{n}, X\right)$, that is, $U^{T} X U=X$. Writing $X=\left[\begin{array}{cc}x_{11} & v^{T} \\ v & \bar{X}\end{array}\right]$, this equation is equivalent to the pair of equations

$$
\bar{U}^{T} v=v \quad \text { and } \quad \bar{U}^{T} \bar{X} \bar{U}=\bar{X} \quad \text { for all } \bar{U} \in \mathcal{O}_{n-1} .
$$

The first equation above implies $v=0$. In the second equation, we can choose $\bar{U}$ so that the left hand side matrix is diagonal, proving that $\bar{X}$ itself must be a diagonal matrix. If $\bar{U}$ is an orthogonal matrix such that $U e_{i}=e_{j}$, then the second equation in (5.1) gives $\bar{X}_{j j}=\left(U e_{i}\right)^{T} \bar{X}\left(U e_{i}\right)=e_{i}^{T} \bar{X} e_{i}=\bar{X}_{i i}$. This proves that $X=\operatorname{diag}(a, b, b, \ldots, b)$ for some $a>0, b>0$.

The following theorem is one of the main results in this paper. As mentioned in the introduction, the first complete published proof of this result appears in [36]. An earlier paper [32] states without proof some of the formulas for the optimal ellipsoids. These ellipsoids are relevant for several versions of the ellipsoid method, see [7] and [13]. The proof below seems to be the first published derivation of these particular optimal ellipsoids using optimization techniques. Perhaps more importantly, the same methodology can be used effectively in many other contexts, as we demonstrate in sections 6 and 7 .

Theorem 5.2. The minimum volume circumscribed ellipsoid $\mathrm{CE}\left(B_{\alpha \beta}\right)$ has the form $E(X, c)$ where $c=\tau e_{1}$ and $X=\operatorname{diag}(a, b, \ldots, b)$, where the parameters $a>0, b>0$, and $\alpha<\tau<\beta$ are given as follows:

(i) If $\alpha \beta \leq-1 / n$, then

$$
\tau=0, \text { and } a=b=1 .
$$

(ii) If $\alpha+\beta=0$ and $\alpha \beta>-1 / n$, then

$$
\tau=0, \quad a=\frac{1}{n \beta^{2}}, \quad b=\frac{n-1}{n\left(1-\beta^{2}\right)} .
$$

(iii) If $\alpha+\beta \neq 0$ and $\alpha \beta>-1 / n$, then

$$
\begin{aligned}
& \tau=\frac{n(\beta+\alpha)^{2}+2(1+\alpha \beta)-\sqrt{\Delta}}{2(n+1)(\beta+\alpha)}, \\
& a=\frac{1}{n(\tau-\alpha)(\beta-\tau)}, \quad b=a\left(1-\frac{2 \tau}{\alpha+\beta}\right),
\end{aligned}
$$

where $\Delta=n^{2}\left(\beta^{2}-\alpha^{2}\right)^{2}+4\left(1-\alpha^{2}\right)\left(1-\beta^{2}\right)$. 
Proof. Lemma 5.1 implies that $X=\operatorname{diag}(a, b, \ldots, b)$ and $c=\tau e_{1}$. Writing $u_{i}=\left(y_{i}, z_{i}\right) \in$ $\mathbb{R} \times \mathbb{R}^{n-1}, i=1, \ldots, k$, where $1=\left\|u_{i}\right\|^{2}=y_{i}^{2}+\left\|z_{i}\right\|^{2}$, that is $\left\|z_{i}\right\|^{2}=1-y_{i}^{2}$, and noting that $\left(u_{i}-c\right)\left(u_{i}-c\right)^{T}=\left[\begin{array}{cc}\left(y_{i}-\tau\right)^{2} & \left(y_{i}-\tau\right) z_{i}^{T} \\ \left(y_{i}-\tau\right) z_{i} & z_{i} z_{i}^{T}\end{array}\right]$, the Fritz John (necessary and sufficient) optimality conditions 2.6 may be written in the form

$$
\begin{aligned}
\tau & =\frac{1}{n} \sum_{i=1}^{k} \lambda_{i} y_{i}, \quad 0=\sum_{i=1}^{k} \lambda_{i} z_{i}, \quad \sum_{i=1}^{k} \lambda_{i}=n, \\
\frac{1}{a} & =\sum_{i=1}^{k} \lambda_{i}\left(y_{i}-\tau\right)^{2}, \quad 0=\sum_{i=1}^{k} \lambda_{i}\left(y_{i}-\tau\right) z_{i}, \quad \frac{1}{b} I_{n-1}=\sum_{i=1}^{k} \lambda_{i} z_{i} z_{i}^{T}, \\
0 & =a\left(y_{i}-\tau\right)^{2}+b\left(1-y_{i}^{2}\right)-1, \quad i=1, \ldots, k, \\
0 & \geq a(y-\tau)^{2}+b\left(1-y^{2}\right)-1, \quad \forall y \in[\alpha, \beta] .
\end{aligned}
$$

The last line expresses the feasibility condition $K \subseteq E(X, c)$ : any point $x=(y, z)$ satisfying $\alpha \leq y \leq \beta$ and $\|x\|=1$ lies in $K$, hence in $E(X, c)$, so that it satisfies the conditions $y^{2}+\|z\|^{2}=1$ and $a(y-\tau)^{2}+b\|z\|^{2} \leq 1$.

The conditions (5.5) -5.8 thus characterize the ellipsoid $\mathrm{CE}(K)$ for $K=B_{\alpha \beta}$ in 4.1. Since the ellipsoid $\mathrm{CE}(K)$ is unique, its parameters $(\tau, a, b)$ are unique and can be recovered from the above conditions. These are done in the technical lemmas below.

Lemma 5.3. If $a=b$ in the ellipsoid $\operatorname{CE}\left(B_{\alpha \beta}\right)$, then $\tau=0, a=b=1$, and $\alpha \beta \leq-1 / n$.

Proof. Since $a=b,(5.7)$ gives the equation $2 a \tau y_{i}=a \tau^{2}+a-1$. We have $\tau=0$, since otherwise all $y_{i}$ are the same, and the first and third equations in 5.5 imply that $y_{i}=\tau$, contradicting the first equation in (5.6). The equation $2 a \tau y_{i}=a \tau^{2}+a-1$ reduces to $a=1=b$. Finally, since $\alpha \leq y_{i} \leq \beta$, we obtain

$$
0 \geq \sum_{i=1}^{k} \lambda_{i}\left(y_{i}-\alpha\right)\left(y_{i}-\beta\right)=\sum_{i=1}^{k} \lambda_{i} y_{i}^{2}-(\alpha+\beta) \sum_{i=1}^{k} \lambda_{i} y_{i}+\alpha \beta \sum_{i=1}^{k} \lambda_{i}=1+n \alpha \beta,
$$

where the last equation follows since $\sum_{i=1}^{k} \lambda_{i} y_{i}^{2}=1$ from the first equation in (5.6) and $\sum_{i=1}^{k} \lambda_{i} y_{i}=0, \sum_{i=1}^{k} \lambda_{i}=n$ from 5.5.

Lemma 5.4. If $a \neq b$ in the ellipsoid $\mathrm{CE}\left(B_{\alpha \beta}\right)$, then $a>b$ and the leading coordinate $y_{i}$ of a contact point must be $\alpha$ or $\beta$, and both values are taken.

Proof. Observe that the function $g(y):=a(y-\tau)^{2}+b\left(1-y^{2}\right)-1$ in 5.8 is nonpositive on the interval $I=[\alpha, \beta]$ and equals zero at each $y_{i}$. We claim that $y_{i}$ can not take a single value: otherwise the first and third equations in (5.5) imply that $y_{i}=\tau$, contradicting the first equation in (5.6). (This result also follows from Corollary 2.3.) Since $g$ is a quadratic function, $y_{i}$ must take exactly two values, and (5.8) implies that these two values must coincide with the endpoints of the interval $I$. Furthermore, $g(y) \leq 0$ only on $I$, has a global minimizer there, and so it must be a strictly convex function. This proves that $a>b$.

Lemma 5.5. If $a \neq b$ in the ellipsoid $\operatorname{CE}\left(B_{\alpha \beta}\right)$, then $(\tau, a, b)$ are given by the equations (5.3) and 5.4. Moreover, $\alpha \beta>-1 / n$. 
Proof. Lemma 5.4 and equation 5.7) give $a(\beta-\tau)^{2}+b\left(1-\beta^{2}\right)=1$ and $a(\alpha-\tau)^{2}+b\left(1-\alpha^{2}\right)=$ 1. Subtracting the second equation from the first and dividing by $\beta-\alpha \neq 0$ yields the equation $\tau=(1-b / a)(\alpha+\beta) / 2$. We also have

$$
\begin{aligned}
0 & =\sum_{i=1}^{k} \lambda_{i}\left(y_{i}-\alpha\right)\left(y_{i}-\beta\right)=\sum_{i=1}^{k} \lambda_{i}\left[\left(y_{i}-\tau\right)-(\alpha-\tau)\right] \cdot\left[\left(y_{i}-\tau\right)-(\beta-\tau)\right] \\
& =\sum_{i=1}^{k} \lambda_{i}\left(y_{i}-\tau\right)^{2}-(\alpha+\beta-2 \tau) \sum_{i=1}^{k} \lambda_{i}\left(y_{i}-\tau\right)+(\alpha-\tau)(\beta-\tau) \sum_{i=1}^{k} \lambda_{i} \\
& =\frac{1}{a}+n(\alpha-\tau)(\beta-\tau),
\end{aligned}
$$

where the first equation follows from Lemma 5.4, the last equation from 5.5 and 5.6 .

Altogether, we have the equations

$$
1=a(\alpha-\tau)^{2}+b\left(1-\alpha^{2}\right), \quad \tau=\left(1-\frac{b}{a}\right) \cdot \frac{\alpha+\beta}{2}, \quad \frac{1}{a}=n(\tau-\alpha)(\beta-\tau),
$$

which we use to compute the variables $a, b$, and $\tau$.

If $\alpha=-\beta$, then the second equation above gives $\tau=0$. Then the third and first equations in (5.9) give $a=1 /\left(n \alpha^{2}\right)$ and $b=(n-1) /\left(n\left(1-\alpha^{2}\right)\right)$, respectively. Lastly, Lemma 5.4 gives $a>b$, and this implies $1+n \alpha \beta>0$.

If $\beta \neq-\alpha$, then the first and third equations in 5.9 give $(\alpha-\tau)^{2}+(b / a)\left(1-\alpha^{2}\right)=$ $n(\tau-\alpha-)(\beta-\tau)$ and the second equation gives $b / a=1-2 \tau /(\alpha+\beta)$. Substituting this value of $b / a$ in the preceding one leads to the quadratic equality for $\tau$,

$$
(n+1)(\alpha+\beta) \tau^{2}-\left(n(\alpha+\beta)^{2}+2(1+\alpha \beta)\right) \tau+(\alpha+\beta)(1+n \alpha \beta)=0 .
$$

A straightforward but tedious calculation shows that the discriminant is $\Delta=n^{2}\left(\beta^{2}-\right.$ $\left.\alpha^{2}\right)^{2}+4\left(1-\beta^{2}\right)\left(1-\alpha^{2}\right)>0$. We claim that the feasible root is the one with negative discriminant. If $\bar{\tau}$ is the root with positive discriminant, then $\bar{\tau}-\beta=\left[n\left(\alpha^{2}-\beta^{2}\right)+2\left(1-\beta^{2}\right)+\right.$ $\sqrt{\Delta}] /(2(n+1)(\alpha+\beta)) \geq 0$. Recalling that $\beta^{2} \geq \alpha^{2}$, we have $\left[n\left(\alpha^{2}-\beta^{2}\right)+2\left(1-\beta^{2}\right)\right]^{2}-\Delta=$ $4(n+1)\left(1-\beta^{2}\right)\left(\alpha^{2}-\beta^{2}\right) \leq 0$. This gives $\bar{\tau} \geq \beta$, proving the claim, as we must have $\alpha<\tau<\beta$. Therefore,

$$
\begin{aligned}
& \tau=\frac{n(\alpha+\beta)^{2}+2(1+\alpha \beta)-\sqrt{\Delta}}{2(n+1)(\alpha+\beta)}, \\
& a=\frac{1}{n(\tau-\alpha)(\beta-\tau)}, \quad b=\frac{1-a(\alpha-\tau)^{2}}{1-\alpha^{2}},
\end{aligned}
$$

where the equations for $a$ and $b$ follow from the first and second equations in 5.9 .

Finally, $\tau=(1-b / a)(\alpha+\beta) / 2$ from (5.9) and Lemma 5.4 gives $a>b$, implying $\tau>0$. From the formula above for $\tau$, we get

$$
0<\left(n(\alpha+\beta)^{2}+2(1+n \alpha \beta)\right)^{2}-\Delta=4(n+1)(\alpha+\beta)^{2}(1+n \alpha \beta) .
$$

This gives $1+n \alpha \beta>0$. The lemma is proved.

Remark 5.6. Some of the results contained in Theorem 5.2 may seem very counterintuitive. For example, consider the slab $B_{\alpha \beta}$ when $-\alpha=\beta=1 / \sqrt{n}$. Although the width 
of this slab is $2 / \sqrt{n}$, very small for large $n$, the optimal covering ellipsoid is the unit ball. This seemingly improbable behavior may be explained by the concentration of measure phenomenon: most of the volume of a high dimensional ball is concentrated in a thin strip around the "equator", see for example [3. There is a sizable literature on concentration of measure; the interested reader may consult the reference 24] for more information on this important topic.

\subsection{Determination of the circumscribed ellipsoid by nonlinear pro- gramming}

In this section, we give a proof of Theorem 5.2 which is completely independent of the previous one based on semi-infinite programming. The proof uses the uniqueness of the covering ellipsoid, Lemma 5.1 on the form of the optimal ellipsoid, and Corollary 2.3. We thus need proofs of the first and the last results that do not depend on the results of 82 and Theorem 5.2. We note that an elementary and direct proof of the uniqueness of the optimal covering ellipsoid can be found, for example, in Danzer et al. [11, and we supply an independent, direct proof of Corollary 2.3, in the spirit of the proof in Grunbaum [15] for the maximum volume inscribed ellipsoid. This last result is not strictly necessary, but it simplifies our proofs, and it may be of independent interest.

Corollary 5.7. Let $K$ be a convex body in $\mathbb{R}^{n}$. The contact points of $\mathrm{CE}(K)$ are not contained in any closed halfspace whose bounding hyperplane passes through the center of $\mathrm{CE}(K)$.

Proof. We assume without loss of generality that the ellipsoid is the unit ball $B_{n}$. Clearly, it suffices to show that the open halfspace $B^{+}:=\left\{x: x_{n}>0\right\}$ contains a point of $E \cap \partial K$. We prove this by contradiction.

Consider the family of ellipsoids $E_{\lambda}:=E(a, b, \lambda)=\left\{x: f(x)=a \sum_{i=1}^{n-1} x_{i}^{2}+b\left(x_{n}+\lambda\right)^{2} \leq\right.$ $1\}$ such that the points $\left\{e_{i}\right\}_{1}^{n-1}$ and $-e_{n}=(0,0, \ldots, 0,-1)$ lie on the boundary of $E_{\lambda}$. We have $b=1 /(1-\lambda)^{2}, a=1-\lambda^{2} /(1-\lambda)^{2}=(1-2 \lambda) /(1-\lambda)^{2}$, and

$$
\operatorname{vol}\left(E_{\lambda}\right)=\left(a^{n-1} b\right)^{-1}=\frac{(1-\lambda)^{2 n}}{(1-2 \lambda)^{n-1}} .
$$

We claim that $K \subseteq E_{\lambda}$ for small enough $\lambda>0$. On the one hand, if $\|x\|=1$ and $x_{n} \leq 0$, we have $f(x)-1=\frac{2 \lambda}{(1-\lambda)^{2}}\left(x_{n}^{2}+x_{n}\right) \leq 0$. On the other hand, since $B^{+}$contains no contact points, there exists $\epsilon>0$ such $\|x\|<1-\epsilon$ for all $x \in K \cap B^{+}$. It follows by continuity that $K \cap B^{+} \subset E_{\lambda}$ for small enough $\lambda>0$. These prove the claim.

Lastly, $\operatorname{vol}\left(E_{\lambda}\right)<1$, since $(1-\lambda)^{2 n}-(1-2 \lambda)^{n-1}=[1-2 n \lambda+o(\lambda)]-[1-(n-1)(-2 \lambda)+$ $o(\lambda)]=-2 \lambda+o(\lambda)<0$ for small $\lambda>0$.

Theorem 5.8. The minimum volume covering ellipsoid problem for the slab $B_{\alpha \beta}$ can be formulated as the nonlinear programming problem

$$
\begin{array}{cl}
\min & -\ln a-(n-1) \ln b, \\
\text { s.t. } \quad & a \tau-\left(\frac{\alpha+\beta}{2}\right)(a-b)=0, \\
& a \tau^{2}+b-1-\alpha \beta(a-b)=0, \\
& -a+b \leq 0,
\end{array}
$$


whose solution is the same as the one given in Theorem 5.2.

Proof. It follows from Lemma 5.1 that the optimal ellipsoid $E(X, c)$ has the form $X=$ $\operatorname{diag}(a, b, \ldots, b)$ and $c=\tau e_{1}$. Thus, the feasibility condition $B_{\alpha \beta} \subseteq E(X, c)$ translates into the condition that the quadratic function

$$
g(u)=a(u-\tau)^{2}+b\left(1-u^{2}\right)-1
$$

is non-positive on the interval $I=[\alpha, \beta]$. Furthermore, Corollary 5.7 implies that there exist at least two contact points, which translates into the condition that the quadratic function $g(u)$ takes the value zero at two distinct points in the interval $I$. A moment's reflection shows that $g(u)$ must take the value zero at the endpoints $\alpha$ and $\beta$. Consequently, the function $g$ is a non-negative multiple of the function $(u-\alpha)(u-\beta)$, that is

$$
g(u)+\mu(u-\alpha)(\beta-u)=0, \quad \text { for some } \quad \mu \geq 0,
$$

giving $a-b=\mu \geq 0,(\alpha+\beta) \mu-2 a \tau=0$, and $a \tau^{2}+b-1-\alpha \beta \mu=0$. If we eliminate $\mu$ from these constraints, we arrive at the optimization problem (5.11). We have for it the Fritz John optimality conditions (for ordinary nonlinear programming)

$$
\begin{aligned}
\lambda_{1}\left(\tau-\frac{\alpha+\beta}{2}\right)+\lambda_{2}\left(\tau^{2}-\alpha \beta\right)-\lambda_{3} & =\frac{\lambda_{0}}{a}, \\
\lambda_{1}\left(\frac{\alpha+\beta}{2}\right)+\lambda_{2}(1+\alpha \beta)+\lambda_{3} & =\frac{\lambda_{0}(n-1)}{b}, \\
\lambda_{1}+2 \lambda_{2} \tau & =0,
\end{aligned}
$$

for some $\left(\lambda_{0}, \lambda_{1}, \lambda_{2}, \lambda_{3}\right) \neq 0, \lambda_{0} \geq 0, \lambda_{3} \geq 0$, and satisfying $\lambda_{3}(a-b)=0$.

Adding the first two equations above and substituting $\lambda_{1}=-2 \lambda_{2} \tau$ from the third equation gives

$$
\lambda_{2}\left(1-\tau^{2}\right)=\frac{\lambda_{0}}{a}+\frac{\lambda_{0}(n-1)}{b} .
$$

If $\lambda_{0}=0$, we would have $\lambda_{2}\left(1-\tau^{2}\right)=0$, and since $\tau \neq \pm 1, \lambda_{2}=0$, and eventually $\lambda_{1}=0=\lambda_{3}$, a contradiction. Thus, we may assume that $\lambda_{0}=1$.

Then the above equation gives

$$
\lambda_{2}\left(1-\tau^{2}\right)=\frac{1}{a}+\frac{n-1}{b} .
$$

We solve for the decision variables $(a, b, \tau)$ discussing separately the cases $a=b$ and $a \neq b$ in the above optimality conditions. If $a=b$, then the first constraint in (5.11) gives $\tau=0$ and the second constraint gives $b=1$. It remains to prove that $\alpha \beta \leq-1 / n$. The equation 5.13) gives $\lambda_{2}=n$, and $\lambda_{1}=-2 \lambda_{2} \tau=0$. Substituting these in the first equation in 5.12 yields $0 \leq \lambda_{3}=-n \alpha \beta-1$, that is, $\alpha \beta \leq-1 / n$.

We now consider the case $a \neq b$ but $\alpha+\beta=0$. The first constraint in problem (5.11) gives $\tau=0$ and the third one gives $\lambda_{3}=0$. Consequently, the first two conditions in (5.12) can be written as $\lambda_{2} \beta^{2} a=1$ and $\lambda_{2}\left(1-\beta^{2}\right) b=n-1$, respectively, and the second constraint in 5.11) gives $\beta^{2} a+\left(1-\beta^{2}\right) b=1$. These imply $\lambda_{2}=n$, and $a=1 / n \beta^{2}, b=\frac{n-1}{n\left(1-\beta^{2}\right)}$. Lastly, the condition $a>b$ gives $\alpha \beta>-1 / n$.

Finally, we treat the case $a>b$ and $\alpha+\beta \neq 0$. Again we have $\lambda_{3}=0$ and 


$$
(n-1) \frac{-\tau^{2}+(\alpha+\beta) \tau-\alpha \beta}{-(\beta+\alpha) \tau+(1+\alpha \beta)}=\frac{b}{a}=\frac{\beta+\alpha-2 \tau}{\beta+\alpha} .
$$

Here the first equality is obtained by dividing the first equation in 5.12 by the second one and substituting $\lambda_{1}=-2 \lambda_{2} \tau$, and the second equality follows from the first constraint in problem (5.11). Consequently, $\tau$ satisfies the quadratic equality

$$
(n+1)(\alpha+\beta) \tau^{2}-\left[n(\alpha+\beta)^{2}+2(1+\alpha \beta)\right] \tau+(\alpha+\beta)(1+n \alpha \beta)=0,
$$

which is the same equation as 5.10$)$ in the proof of Lemma 5.5. Following similar arguments, we find that

$$
\tau=\frac{n(\alpha+\beta)^{2}+2(1+\alpha \beta)-\sqrt{\Delta}}{2(n+1)(\alpha+\beta)},
$$

where $\Delta$ is the discriminant of the quadratic equation (5.14). Solving the first and the second constraints in 5.11 for $a$, say by Cramer's rule, we find

$$
a=\frac{\alpha+\beta}{(\alpha+\beta)\left(\tau^{2}+1\right)-2 \tau(1+\alpha \beta)} .
$$

It follows from (5.14) that the denominator on the right hand side of the expression above equals

$$
n(\alpha+\beta)^{2} \tau-n(\alpha+\beta) \tau^{2}-n(\alpha+\beta) \alpha \beta=n(\alpha+\beta)(\tau-\alpha)(\beta-\tau) .
$$

This gives

$$
a=\frac{1}{n(\tau-\alpha)(\beta-\tau)}, \quad \text { and } \quad b=\frac{\alpha+\beta-2 \tau}{\alpha+\beta} a .
$$

Finally, the inequality $\alpha \beta>-1 / n$ follows from the same argument at the end of the proof of Lemma 5.5

\section{Determination of the inscribed ellipsoid of a slab}

In this section, we give explicit formulae for the maximum volume inscribed ellipsoid of the slab $B_{\alpha \beta}$ in (4.1) using a semi-infinite programming approach. Without any loss of generality, we again assume throughout this section that $\beta^{2} \geq \alpha^{2}$.

It is convenient to set up this problem as the semi-infinite program

$$
\min \left\{-\ln \operatorname{det}(A): A y+c \in B_{\alpha \beta}, \quad \forall y:\|y\|=1\right\},
$$

in which we represent the inscribed ellipsoid as $E=c+A\left(B_{n}\right)$ where $A$ is a symmetric, positive definite matrix with $\operatorname{vol}(E)=\omega_{n} \operatorname{det} A$. Lemma 5.1 implies that the optimal ellipsoid has the form $A=\operatorname{diag}(a, b, \ldots, b)$ and $c=\tau e_{1}$ for some $a>0, b>0$, and $\tau$ in $\mathbb{R}$. Writing $y=(u, z)$ in $\mathbb{R} \times \mathbb{R}^{n-1}$, we can replace the above semi-infinite program with a simpler one

$$
\begin{array}{ll}
\min & -\ln a-(n-1) \ln b, \\
\text { s.t. } & -a u-\tau \leq-\alpha, \\
& a u+\tau \leq \beta, \\
& (a u+\tau)^{2}+b^{2}\left(1-u^{2}\right) \leq 1,
\end{array} \quad \forall u \in[-1,1]
$$


in which the decision variables are $(a, b, \tau)$ and the index set is $[-1,1]$.

We make some useful observations before writing down the optimality conditions for Problem 6.1. Theorem 2.1 implies that the optimality conditions will involve at most three active constraints with corresponding multipliers positive. Clearly, the first constraint above can be active only for $u=-1$ and the second one for $u=1$.

Next, if $\alpha>-1$, we claim that the third constraint is active for at most one index value $u$. Otherwise, the quadratic function

$$
g(u):=(a u+\tau)^{2}+b^{2}\left(1-u^{2}\right)-1
$$

is non-positive on the interval $[-1,1]$ and equals zero for two distinct values of $u$. If the function $g(u)$ is actually a linear function $(\mathrm{a}=\mathrm{b})$, then it is identically zero; otherwise, it is easy to see that $g(u)$ must be equal to zero at the endpoints -1 and 1 . In all cases, we have $g(-1)=g(1)=0$, so that $(-a+\tau)^{2}=1=(a+\tau)^{2}$. This gives $a-\tau=1=a+\tau$, since the other possibilities give $a=0$ or $a=-1$. But then $a=1, \tau=0$, and $1=a-\tau \leq-\alpha$, where the inequality expresses the feasibility of the first inequality in Problem 6.1. We obtain $\alpha=-1$ and $\beta=1$, a contradiction. The claim is proved.

The following theorem is another major result of this paper.

Theorem 6.1. The maximal inscribed ellipsoid $\operatorname{IE}\left(B_{\alpha \beta}\right)$ has the form $E=c+A\left(B_{n}\right)$ where $c=\tau e_{1}, \alpha<\tau<\beta, A=\operatorname{diag}(a, b, \ldots, b)$ with $a>0, b>0$, satisfying the following conditions:

(i) If $\alpha=-\beta$, then

$$
\tau=0, \quad a=\beta, \quad b=1 .
$$

(ii) If $4 n\left(1-\alpha^{2}\right)<(n+1)^{2}\left(\beta^{2}-\alpha^{2}\right)$, then

$$
\begin{aligned}
& \tau=\frac{\alpha+\sqrt{\alpha^{2}+4 n\left(1-\alpha^{2}\right) /(n+1)^{2}}}{2}, \\
& a=\tau-\alpha, \quad b^{2}=a(a+n \tau),
\end{aligned}
$$

(iii) If $4 n\left(1-\alpha^{2}\right) \geq(n+1)^{2}\left(\beta^{2}-\alpha^{2}\right)$ and $\alpha \neq-\beta$, then

$$
\begin{aligned}
\tau & =\frac{\beta+\alpha}{2}, \quad a=\frac{\beta-\alpha}{2}, \\
b^{2} & =a^{2}+\left(\frac{\beta^{2}-\alpha^{2}}{2\left(\sqrt{1-\alpha^{2}}-\sqrt{1-\beta^{2}}\right)}\right)^{2} .
\end{aligned}
$$

Proof. If $\alpha=-1$, then $\beta=1$ and the optimal ellipsoid is the unit ball $B_{n}$, which agrees with (i) of the theorem. We assume in the rest of the proof that $\alpha>-1$.

We saw above that each of the constraints in 6.1 can be active for at most one value of $u$ in $[-1,1]$, and the first and second constraints for $u=-1$ and $u=1$, respectively. Then Theorem 2.1 gives the optimality conditions

$$
\begin{aligned}
\lambda_{1}+\lambda_{2}+\delta(a u+\tau) u & =\frac{\lambda_{0}}{a}, \\
\delta b\left(1-u^{2}\right) & =\frac{(n-1) \lambda_{0}}{b}, \quad u \in[-1,1], \\
-\lambda_{1}+\lambda_{2}+\delta(a u+\tau) & =0,
\end{aligned}
$$


where the non-negative multipliers $\left(\lambda_{0}, \lambda_{1}, \lambda_{2}, \delta / 2\right) \neq 0$ correspond to the objective function, and the first, second, and third (active) constraints in 6.1. respectively.

Our first claim is that $\lambda_{0}>0$. Otherwise, the second equation in 6.5 gives $\delta=0$ or $u= \pm 1$. If $\delta=0$, then the first equation gives the contradiction $\lambda_{1}=\lambda_{2}=0$. If $u=-1$, then we have $\pm 1=a-\tau \leq-\alpha<1$, implying $a-\tau=-1$ or $\tau=a+1>1$, another contradiction. If $u=1$, we have $\pm 1=a+\tau$. If $a+\tau=1$, then the first equation in 6.5 gives $\lambda_{1}+\lambda_{2}+\delta=0$, that is, $\lambda_{1}=\lambda_{2}=\delta=0$, a contradiction. If $a+\tau=-1$, then $\tau=-a-1<-1$, yet another contradiction. The claim is proved. We set $\lambda_{0}=1$.

The second equation in 6.5 gives $\delta>0$, and that $g(u)=0$ for some $u$ in $(-1,1)$ and negative elsewhere on $[-1,1]$. Note this means that $u$ is the global maximum as well as the unique root of $g$ on $\mathbb{R}$, leading to the conditions

$$
b>a, \quad u=\frac{a \tau}{b^{2}-a^{2}}, \quad b^{2} \tau^{2}=\left(1-b^{2}\right)\left(b^{2}-a^{2}\right),
$$

where the last equation expresses the fact that the discriminant of $g$ equals zero. We also have

$$
a u+\tau=a \frac{a \tau}{b^{2}-a^{2}}+\tau=\frac{b^{2} \tau}{b^{2}-a^{2}} .
$$

Our second claim is that

$$
-a+\tau=\alpha .
$$

If not, then $-a+\tau>\alpha, \lambda_{1}=0$, and the third equation in (6.5) gives $\lambda_{2}=-\delta(a u+\tau)$. Substituting this into the first equation in (6.5) leads to $\delta(a u+\tau)(u-1)=a^{-1}$, and consequently to $a u+\tau<0$. But then $\lambda_{2}>0$ and $a+\tau=\beta$, which together with $-a+\tau>\alpha$ gives $\tau>(\alpha+\beta) / 2 \geq 0$, that is, $\tau>0$. Moreover, the second equation in (6.6) gives $u>0$, and this leads to the contradiction that $-\lambda_{2}=\delta(a u+\tau)>0$. The claim is proved.

Next, we have

$$
\begin{aligned}
\frac{1}{a}-2 \lambda_{2} & =\delta(a u+\tau)(u+1)=\frac{n-1}{b^{2}\left(1-u^{2}\right)} \cdot \frac{b^{2} \tau}{b^{2}-a^{2}}(u+1) \\
& =\frac{(n-1) \tau}{(1-u)\left(b^{2}-a^{2}\right)}=\frac{(n-1) u}{a(1-u)},
\end{aligned}
$$

where the first equality is obtained by adding the first and third equations in (6.5), the second equality follows by substituting the value of $\delta$ from the second equation in (6.5) and the value of $a u+\tau$ from (6.7), and the last equality follows by substituting the value $\tau /\left(b^{2}-a^{2}\right)=u / a$ from the first equation in 6.6$)$. Therefore,

$$
\lambda_{2}=\frac{1-n u}{2 a(1-u)} .
$$

Consequently, $u \leq 1 / n$ and $u=1 / n$ if and only if $\lambda_{2}=0$. Furthermore, we have $u \geq 0$ : if $u<0$, then $\tau<0$ by virtue of the first equation in (6.6), so that $a u+\tau<0$. But then the third equation in (6.5) gives $\lambda_{2}>\lambda_{1} \geq 0$, implying $a+\tau=\beta$. This and (6.8) give $\tau=(\alpha+\beta) \geq 0$, a contradiction. Therefore,

$$
0 \leq u \leq \frac{1}{n}, \quad \text { and } \quad u=\frac{1}{n} \quad \Longleftrightarrow \quad \lambda_{2}=0 .
$$

We can now prove part (i) of the theorem. We first show that 


$$
u=0 \quad \Longleftrightarrow \quad \alpha=-\beta
$$

If $\alpha=-\beta$, then Lemma 5.1 implies that $\tau=0$, which in turn implies $u=0$. Conversely, if $u=0$, then $\tau=0$ and $\lambda_{2}>0$, and we have $-a+\tau=\alpha$ and $a+\tau=\beta$, leading to $0=\tau=(\beta+\alpha) / 2$. Consequently, (6.8) gives $a=-\alpha=\beta$ and the second equation in (6.6) gives $b=1$.

We now consider the remaining cases $0<u \leq 1 / n$. We note that

$$
(a u+\tau) u \tau=\frac{u\left(b^{2} \tau^{2}\right)}{b^{2}-a^{2}}=u\left(1-b^{2}\right)=u\left(1-a^{2}\right)-a \tau,
$$

where the first equality follows from (6.7) and last two equalities from (6.6), leading to a quadratic equation for $u$,

$$
(a \tau) u^{2}-\left(1-a^{2}-\tau^{2}\right) u+a \tau=0
$$

Define $\varepsilon \geq 0$ such that $a+\tau=\beta-\varepsilon=: \beta_{\varepsilon}$. The equation $a+\tau=\beta_{\varepsilon}$ together with equation $-a+\tau=\alpha$ from 6.8 give

$$
\tau=\frac{\beta_{\varepsilon}+\alpha}{2}>0, \quad a=\frac{\beta_{\varepsilon}-\alpha}{2}>0 .
$$

Substituting these in 6.10 gives another quadratic equality for $u$,

$$
\left(\beta_{\varepsilon}^{2}-\alpha^{2}\right) u^{2}-2\left(2-\beta_{\varepsilon}^{2}-\alpha^{2}\right) u+\left(\beta_{\varepsilon}^{2}-\alpha^{2}\right)=0 .
$$

It is easy to verify, using 6.9 , that

$$
\begin{aligned}
& \lambda_{2}>0 \quad \Longleftrightarrow 0<u<\frac{1}{n} \quad \Longleftrightarrow \quad(n+1)^{2}\left(\beta_{\varepsilon}^{2}-\alpha^{2}\right)<4 n\left(1-\alpha^{2}\right), \\
& \lambda_{2}=0 \quad \Longleftrightarrow u=\frac{1}{n} \quad \Longleftrightarrow \quad(n+1)^{2}\left(\beta_{\varepsilon}^{2}-\alpha^{2}\right)=4 n\left(1-\alpha^{2}\right) .
\end{aligned}
$$

Here the second equivalence on the first line follows because the quadratic equation for $u$ in 6.12 has negative value at $u=1 / n$. Since the leading term of the quadratic function is positive, its two roots $r_{1}<r_{2}$ are positive, their product is $1,0<r_{1}<1 / n$, and $r_{2}>n$.

We now make our third and important claim that

$$
a+\tau<\beta \quad \text { iff } \quad 4 n\left(1-\alpha^{2}\right)<(n+1)\left(\beta^{2}-\alpha^{2}\right)
$$

Since $a+\tau \leq \beta$, we consider separately the cases $a+\tau=\beta$ and $a+\tau<\beta$. In the first case, $\varepsilon=0$ and 6.13 gives $(n+1)\left(\beta^{2}-\alpha^{2}\right)<4 n\left(1-\alpha^{2}\right)$ or $(n+1)\left(\beta^{2}-\alpha^{2}\right)=$ $4 n\left(1-\alpha^{2}\right)$, depending on whether $\lambda_{2}>0$ or $\lambda_{2}=0$, respectively. In either situation, we have $(n+1)\left(\beta^{2}-\alpha^{2}\right) \leq 4 n\left(1-\alpha^{2}\right)$. In the second case, we have $\lambda_{2}=0, \varepsilon>0$, and 6.13 gives $(n+1)\left(\beta_{\varepsilon}^{2}-\alpha^{2}\right)=4 n\left(1-\alpha^{2}\right)$, that is, $4 n\left(1-\alpha^{2}\right)<(n+1)\left(\beta^{2}-\alpha^{2}\right)$. The claim is proved.

The computation of the decision variables $(a, b, \tau)$ in the cases (ii) and (iii) now becomes a routine matter. If $4 n\left(1-\alpha^{2}\right)<(n+1)^{2}\left(\beta^{2}-\alpha^{2}\right)$, then 6.14 implies $a+\tau<\beta$, and we have $\lambda_{2}=0, u=1 / n$. Substituting the value $a=\tau-\alpha$ from 6.8 into 6.10) gives the quadratic equation for $\tau$,

$$
(n+1)^{2} \tau^{2}-(n+1)^{2} \alpha \tau-n\left(1-\alpha^{2}\right)=0 .
$$


Since $\tau>0$, the feasible root is given by

$$
\tau=\left(\alpha+\sqrt{\alpha^{2}+4 n\left(1-\alpha^{2}\right) /(n+1)^{2}}\right) / 2 .
$$

Then (6.8) gives $a=\tau-\alpha$ and (6.6) gives $1 / n=u=(a \tau) /\left(b^{2}-a^{2}\right)$, that is, $b^{2}=a^{2}+n a \tau$.

If $4 n\left(1-\alpha^{2}\right) \geq(n+1)^{2}\left(\beta^{2}-\alpha^{2}\right)$, then 6.14 and 6.12 give

$$
u=\frac{\left(\sqrt{1-\alpha^{2}} \pm \sqrt{1-\beta^{2}}\right)^{2}}{\beta^{2}-\alpha^{2}} .
$$

It is easy to verify that the condition $u<1$ is equivalent to $\sqrt{1-\beta^{2}} \pm \sqrt{1-\alpha^{2}}<0$, which is impossible if we choose the plus sign. Thus the feasible root is the one with the negative sign. Finally, the first equation in (6.6) gives $b^{2}=a^{2}+(a \tau) / u$, or more explicitly the formula for $b^{2}$ in 6.4.

We end this section by reducing the semi-infinite program (6.1) to an ordinary nonlinear programming problem. However, we do not attempt to solve the resulting program in order to save space. As we already noted, the linear constraints, the first two inequalities in problem 6.1 , simply reduce to the constraints $a-\tau \leq-\alpha$ and $a+\tau \leq \beta$. In order to reduce the quadratic inequality system to a set of ordinary inequalities, we use a theorem of Lukács characterizing the class of non-negative polynomials on a given interval. A simple inductive proof of Lukács's Theorem can be found in [9]. For a quadratic polynomial $q(u)$ on the interval $I=[a, b]$, this theorem states that $q$ is non-negative on $I$ if and only if there exist scalars $\alpha, \beta$, and $\gamma \geq 0$ such that

$$
q(u)=(\alpha u+\beta)^{2}+\gamma(u-a)(b-u) .
$$

Since the proof is short and simple in this case, we give it, following [9]. Note that the polynomial $p(u):=q(u)-l(u)^{2}$, where $l(u)=[\sqrt{q(a)}(u-b)+\sqrt{q(b)}(u-a)] /(b-a)$, satisfies $p(a)=0=p(b)$, so that there exists a constant $\gamma$ such that $p(u)=\gamma(u-a)(b-u)$. Clearly, 6.15 holds true if we can show that $\gamma \geq 0$. Note that $l(a) \leq 0$ and $l(b) \geq 0$ so that $l(u)=$ $d(u-r)$ for some constant $d$ and $r \in[a, b]$. We have $0 \leq q(u)=d^{2}(u-r)^{2}+\gamma(u-a)(b-u)$, or

$$
-d^{2}(u-r)^{2} \leq \gamma(u-a)(b-u), \quad \forall u \in[a, b] .
$$

If $r$ is in $(a, b)$, then choosing $u=r$ gives $\gamma \geq 0$. If $r=a$ or $r=b$, then choosing $u$ near $r$ gives $\gamma \geq 0$. This completes the proof.

We remark that the result we just proved also follows from the one dimensional case of the S-procedure, see [29] or [28].

Applying this result to our quadratic function $-q(u)=-(a u+\tau)^{2}-b^{2}\left(1-u^{2}\right)+1$ which is non-negative on the interval $[-1,1]$, we see that there exists scalars $c, d$, and $\gamma \geq 0$ such that

$$
(a u+\tau)^{2}+b^{2}\left(1-u^{2}\right)-1=-(c u+d)^{2}+\gamma\left(u^{2}-1\right),
$$

that is, $a^{2}-b^{2}+c^{2}-\gamma=0, a \tau+c d=0$, and $b^{2}+\tau^{2}+d^{2}+\gamma=1$. Therefore, the problem of finding $\operatorname{IE}\left(B_{\alpha \beta}\right)$ reduces to the nonlinear programming problem 


$$
\begin{array}{cl}
\min & -\ln a-(n-1) \ln b, \\
\text { s.t. } & -a+\tau \geq \alpha, \\
& a+\tau \leq \beta \\
& a^{2}-b^{2}+c^{2}-\gamma=0, \\
& a \tau+c d=0 \\
& b^{2}+\tau^{2}+d^{2}+\gamma=1, \\
& \gamma \geq 0
\end{array}
$$

in which the decision variables are $(a, b, \tau, c, d, \gamma)$ and $\alpha, \beta$ are parameters.

\section{Determination of the circumscribed ellipsoid of a truncated second-order cone or a cylinder}

In this section, one of the problems we are interested in is finding the minimum volume ellipsoid covering the truncated second-order cone

$$
K=\left\{x=\left(x_{1}, \bar{x}\right) \in \mathbb{R} \times \mathbb{R}^{n-1}:\|B(\bar{x}-c)\| \leq x_{1}, a \leq x_{1} \leq b\right\},
$$

where $B$ is an invertible matrix in $\mathbb{R}^{(n-1) \times(n-1)}$ and $0 \leq a<b$ are constants. By an affine change of $\bar{x}$, we may assume that $c=0$ and $B=I_{n-1}$. We claim that by further affine change of variables, we can reduce the convex body $K$ to have the form

$$
Q_{\alpha \beta}:=\operatorname{conv}\left(S_{\alpha} \cup S_{\beta}\right),
$$

where $S_{\alpha}:=\left\{x \in B_{n}: x_{1}=\alpha\right\}, S_{\beta}:=\left\{x \in B_{n}: x_{1}=\beta\right\}$, and $-1 \leq \alpha<\beta \leq 1$. Consider the ball $B$ in $\mathbb{R}^{n}$ with center $(a+b) e_{1}$ and radius $\sqrt{a^{2}+b^{2}}$. The slice $P_{a}:=\left\{(a, \bar{x}) \in \mathbb{R}^{n}\right.$ : $\|\bar{x}\| \leq a\} \subset K$ lies in $B$, since $\|(a, \bar{x})-(a+b, 0)\|^{2}=b^{2}+\|\bar{x}\|^{2} \leq a^{2}+b^{2}$, and similarly $P_{b} \subset B$. A further translation and then scaling transforms $B$ into $B_{n}$. This proves the claim. Conversely, if $-\alpha \neq \beta, Q_{\alpha \beta}$ can be viewed as a truncated second-order cone.

The other problem we are interested in is finding the minimum volume ellipsoid covering a cylinder. If $-\alpha=\beta$, then $Q_{\alpha \beta}$ is clearly a cylinder. Conversely, any cylinder can be transformed into such a $Q_{\alpha \beta}$ by an affine transformation. Consequently, the $\operatorname{CE}\left(Q_{\alpha \beta}\right)$ problem formulates both problems at the same time.

Theorem 7.1. The ellipsoid $\operatorname{CE}\left(Q_{\alpha \beta}\right)$ has the form $E(X, c)$ where $c=\tau e_{1}$ and $X=$ $\operatorname{diag}(a, b, \ldots, b)$, where the parameters $a>0, b>0$, and $\alpha<\tau<\beta$ are given as follows:

(i) If $\alpha \beta=-1 / n$, then

$$
\tau=0, \text { and } a=b=1 .
$$

(ii) If $\alpha+\beta=0$ and $\alpha \beta \neq-1 / n$, then

$$
\tau=0, \quad a=\frac{1}{n \beta^{2}}, \quad b=\frac{n-1}{n\left(1-\beta^{2}\right)} .
$$

(iii) If $\alpha+\beta \neq 0$ and $\alpha \beta \neq-1 / n$, then

$$
\begin{aligned}
& \tau=\frac{n(\beta+\alpha)^{2}+2(1+\alpha \beta)-\sqrt{\Delta}}{2(n+1)(\beta+\alpha)}, \\
& a=\frac{1}{n(\tau-\alpha)(\beta-\tau)}, \quad b=\frac{1-a(\tau-\alpha)^{2}}{1-\alpha^{2}},
\end{aligned}
$$


where $\Delta=n^{2}\left(\beta^{2}-\alpha^{2}\right)^{2}+4\left(1-\alpha^{2}\right)\left(1-\beta^{2}\right)$.

Proof. It is clear that $\operatorname{Aut}\left(Q_{\alpha \beta}\right) \supseteq \operatorname{Aut}\left(B_{\alpha \beta}\right)$, so that Lemma 5.1 $\operatorname{implies} X=\operatorname{diag}(a, b, \ldots, b)$ and $c=\tau e_{1}$ with $a>0, b>0$ and $\tau \in \mathbb{R}$. Let $\left\{u_{i}\right\}_{i=1}^{k}$ be the contact points of the optimal ellipsoid with $Q_{\alpha \beta}$. Writing $u_{i}=\left(y_{i}, z_{i}\right) \in \mathbb{R} \times \mathbb{R}^{n-1}$, we have that $y_{i}$ is either $\alpha$ or $\beta$, and $1=$ $\left\|u_{i}\right\|^{2}=y_{i}^{2}+\left\|z_{i}\right\|^{2}$, that is, $\left\|z_{i}\right\|^{2}=1-y_{i}^{2}$. Since $\left(u_{i}-c\right)\left(u_{i}-c\right)^{T}=\left[\begin{array}{cc}\left(y_{i}-\tau\right)^{2} & \left(y_{i}-\tau\right) z_{i}^{T} \\ \left(y_{i}-\tau\right) z_{i} & z_{i} z_{i}^{T}\end{array}\right]$, Theorem 2.2 yields the following optimality conditions:

$$
\begin{aligned}
\tau & =\frac{1}{n} \sum_{i=1}^{k} \lambda_{i} y_{i}, \quad 0=\sum_{i=1}^{k} \lambda_{i} z_{i}, \quad \sum_{i=1}^{k} \lambda_{i}=n, \\
\frac{1}{a} & =\sum_{i=1}^{k} \lambda_{i}\left(y_{i}-\tau\right)^{2}, \quad 0=\sum_{i=1}^{k} \lambda_{i}\left(y_{i}-\tau\right) z_{i}, \quad \frac{1}{b} I_{n-1}=\sum_{i=1}^{k} \lambda_{i} z_{i} z_{i}^{T}, \\
0 & =a\left(y_{i}-\tau\right)^{2}+b\left(1-y_{i}^{2}\right)-1, \quad i=1, \ldots, k, \\
0 & \geq a(y-\tau)^{2}+b\left(1-y^{2}\right)-1, \quad \text { for } y \in\{\alpha, \beta\} .
\end{aligned}
$$

Here the last line expresses the feasibility condition $Q_{\alpha \beta} \subseteq E(X, c)$, since $Q_{\alpha \beta} \subseteq E(X, c)$ if and only if $\partial S_{\alpha} \cup \partial S_{\beta} \subseteq E(X, c)$.

The conditions (7.4 $-(7.7)$ characterize the ellipsoid $\operatorname{CE}\left(Q_{\alpha \beta}\right)$. Since the ellipsoid is unique, its parameters $(a, b, \tau)$ are unique and can be recovered from the above conditions.

The same arguments in Lemma 5.5 applies here and gives the equation 5.9 ,

$$
1=a(\alpha-\tau)^{2}+b\left(1-\alpha^{2}\right), \quad \tau=\left(1-\frac{b}{a}\right) \cdot \frac{\alpha+\beta}{2}, \quad \frac{1}{a}=n(\tau-\alpha)(\beta-\tau),
$$

which we again use to compute the variables $a, b$, and $\tau$. If $a=b$ in the optimal ellipsoid, (7.8) immediately gives $\tau=0, a=b=1$, and $\alpha \beta=-1 / n$.

Next, if $a \neq b$ and $\alpha=-\beta$, then (7.8) gives $\tau=0, a=1 /\left(n \alpha^{2}\right)$ and $b=(n-$ 1) $/\left(n\left(1-\alpha^{2}\right)\right)$. Furthermore, if we have $a=1=n \alpha^{2}$, then we obtain a contradiction since $b=(n-1) /(n-1)=1=a$. Therefore, $a \neq 1$ and the last equation in $(7.8)$ gives $\alpha \beta \neq-1 / n$.

Lastly, $a \neq b$ and $\alpha \neq-\beta$, then the same argument in Lemma 5.5 gives the quadratic equation 5.10 for $\tau$,

$$
(n+1)(\alpha+\beta) \tau^{2}-\left(n(\alpha+\beta)^{2}+2(1+\alpha \beta)\right) \tau+(\alpha+\beta)(1+n \alpha \beta)=0,
$$

and the resulting equations in 7.3 for $(a, b, \tau)$. Since the middle equation in 7.8$)$ implies $\tau \neq 0$, we have

$$
0 \neq\left(n(\alpha+\beta)^{2}+2(1+n \alpha \beta)\right)^{2}-\Delta=4(n+1)(\alpha+\beta)^{2}(1+n \alpha \beta),
$$

that is, $\alpha \beta \neq-1 / n$.

\section{References}

[1] S. D Ahipaşaoğlu. Solving ellisoidal inclusion and optimal experimental design problems: theory and algorithms. PhD thesis, Cornell University, Ithaca, New York, August 2009. 
[2] S. D. Ahipaşaoğlu, P. Sun, and M. J. Todd. Linear convergence of a modified FrankWolfe algorithm for computing minimum-volume enclosing ellipsoids. Optim. Methods Softw., 23(1):5-19, 2008.

[3] K. Ball. An elementary introduction to modern convex geometry. In Flavors of geometry, volume 31 of Math. Sci. Res. Inst. Publ., pages 1-58. Cambridge Univ. Press, Cambridge, 1997.

[4] E. R. Barnes and A. C. Moretti. Some results on centers of polytopes. Optim. Methods Softw., 20(1):9-24, 2005.

[5] A. Barvinok and G. Blekherman. Convex geometry of orbits. In Combinatorial and computational geometry, volume 52 of Math. Sci. Res. Inst. Publ., pages 51-77. Cambridge Univ. Press, Cambridge, 2005.

[6] F. Behrend. Über die kleinste umbeschriebene und die größte einbeschriebene Ellipse eines konvexen Bereichs. Math. Ann., 115(1):379-411, 1938.

[7] R. G. Bland, D. Goldfarb, and M. J. Todd. The ellipsoid method: a survey. Oper. Res., 29(6):1039-1091, 1981.

[8] G. Blekherman. Convexity properties of the cone of nonnegative polynomials. Discrete Comput. Geom., 32(3):345-371, 2004.

[9] L. Brickman and L. Steinberg. Classroom notes: on nonnegative polynomials. Amer. Math. Monthly, 69(3):218-221, 1962.

[10] L. Danzer, B. Grünbaum, and V. Klee. Helly's theorem and its relatives. In Proc. Sympos. Pure Math., Vol. VII, pages 101-180. Amer. Math. Soc., Providence, R.I., 1963.

[11] L. Danzer, D. Laugwitz, and H. Lenz. Über das Löwnersche Ellipsoid und sein Analogon unter den einem Eikörper einbeschriebenen Ellipsoiden. Arch. Math., 8:214-219, 1957.

[12] V. V. Fedorov. Theory of optimal experiments. Academic Press, New York, 1972. Probability and Mathematical Statistics, No. 12.

[13] M. Grötschel, L. Lovász, and A. Schrijver. Geometric algorithms and combinatorial optimization, volume 2 of Algorithms and Combinatorics. Springer-Verlag, Berlin, second edition, 1993.

[14] P. M. Gruber. Minimal ellipsoids and their duals. Rend. Circ. Mat. Palermo (2), 37(1):35-64, 1988.

[15] B. Grünbaum. Fixing systems and inner illumination. Acta Math. Acad. Sci. Hungar, 15:161-163, 1964.

[16] O. Güler. Foundations of optimization, volume 258 of Graduate Texts in Mathematics. Springer, New York, 2010.

[17] F. Gürtuna. Duality of ellipsoidal approximations via semi-infinite programming. SIAM J. Optim., 20(3):1421-1438, 2009.

[18] F. John. Extremum problems with inequalities as subsidiary conditions. In Studies and Essays Presented to R. Courant on his 60th Birthday, January 8, 1948, pages 187-204. Interscience Publishers, Inc., New York, N. Y., 1948. 
[19] L. G. Khachiyan. A polynomial algorithm in linear programming. Dokl. Akad. Nauk SSSR, 244(5):1093-1096, 1979.

[20] L. G. Khachiyan and M. J. Todd. On the complexity of approximating the maximal inscribed ellipsoid for a polytope. Math. Programming, 61(2, Ser. A):137-159, 1993.

[21] A. W. Knapp. Lie groups beyond an introduction, volume 140 of Progress in Mathematics. Birkhäuser Boston Inc., Boston, MA, second edition, 2002.

[22] H. König and D. Pallaschke. On Khachiyan's algorithm and minimal ellipsoids. Numer. Math., 36(2):211-223, 1980/81.

[23] D. Laugwitz. Differential and Riemannian geometry. Academic Press, New York, 1965.

[24] M. Ledoux. The concentration of measure phenomenon, volume 89 of Mathematical Surveys and Monographs. American Mathematical Society, Providence, RI, 2001.

[25] R. D.C. Monteiro, J. W. O'Neal, and A. S. Nemirovski. A new conjugate gradient algorithm incorporating adaptive ellipsoid preconditioning. Working Paper, 2004.

[26] A. L. Onishchik and È. B. Vinberg. Lie groups and algebraic groups. Springer Series in Soviet Mathematics. Springer-Verlag, Berlin, 1990.

[27] G. Pisier. The volume of convex bodies and Banach space geometry, volume 94 of Cambridge Tracts in Mathematics. Cambridge University Press, Cambridge, 1989.

[28] I. Pólik and T. Terlaky. A survey of the S-lemma. SIAM Review, 49(3):371-418, 2007.

[29] B. T. Polyak. Convexity of quadratic transformations and its use in control and optimization. J. Optim. Theory Appl., 99(3):553-583, 1998.

[30] R. T. Rockafellar. Convex analysis. Princeton Landmarks in Mathematics. Princeton University Press, Princeton, NJ, 1997. Reprint of the 1970 original, Princeton Paperbacks.

[31] M. Rudelson. Contact points of convex bodies. Israel J. Math., 101:93-124, 1997.

[32] N. Z. Shor and V. I. Gershovich. Family of algorithms for solving convex programming problems. Cybernetics, 15:502-508, 1979.

[33] P. Sun and R. M. Freund. Computation of minimum-volume covering ellipsoids. Oper. Res., 52(5):690-706, 2004.

[34] S. P. Tarasov, L. G. Khachiyan, and I. I. Èrlikh. The method of inscribed ellipsoids. Dokl. Akad. Nauk SSSR, 298(5):1081-1085, 1988.

[35] D. M. Titterington. Optimal design: some geometrical aspects of $D$-optimality. Biometrika, 62(2):313-320, 1975.

[36] M. J. Todd. On minimum volume ellipsoids containing part of a given ellipsoid. Math. Oper. Res., 7(2):253-261, 1982.

[37] H. P. Wynn. Results in the theory and construction of $D$-optimum experimental designs. J. Roy. Statist. Soc. Ser. B, 34:133-147, 170-186, 1972. With discussion by M. J. Box, P. Whittle, S. D. Silvey, A. A. Greenfield, Agnes M. Herzberg, J. Kiefer, D. R. Cox, Lynda V. White, A. C. Atkinson, R. J. Brooks, Corwin L. Atwood, and Robin Sibson, and replies by Henry P. Wynn and P. J. Laycock. 
[38] E. A. Yıldırım. On the minimum volume covering ellipsoid of ellipsoids. SIAM J. Optim., 17(3):621-641, 2006.

[39] V. L. Zaguskin. Circumscribed and inscribed ellipsoids of extremal volume. Uspehi Mat. Nauk, 13(6 (84)):89-93, 1958.

[40] Y. Zhang and L. Gao. On numerical solution of the maximum volume ellipsoid problem. SIAM J. Optim., 14(1):53-76, 2003. 